\title{
FULLY NUCLEAR AND COMPLETELY NUCLEAR OPERATORS WITH APPLICATIONS TO $\mathscr{L}_{1}-\mathrm{AND} \mathscr{L}_{\infty}$-SPACES
}

\author{
BY \\ C. P. STEGALL $\left({ }^{1}\right)$ AND J. R. RETHERFORD $\left({ }^{2}\right)$
}

\begin{abstract}
This paper is devoted to a study of the conjecture of A. Grothendieck that if $E$ and $F$ are Banach spaces and all operators from $E$ to $F$ are nuclear, then $E$ or $F$ must be finite dimensional. Two partial solutions are given to this conjecture (Chapters II and IV). In these chapters, operators we call fully nuclear and completely nuclear are introduced and studied. The principal result of these two chapters is that if $\mathscr{L}(E, F)=\mathrm{FN}(E, F)$ or $\mathscr{L}(E, F)=\mathrm{CN}(E, F)$ (and $E$ is isomorphic to a conjugate space or $E^{\prime}$ contains a reflexive subspace in the latter case) then one of $E, F$ is finite dimensional.

Two new properties of Banach spaces are introduced in Chapter I. We call these properties "sufficiently Euclidean" and "the two-series property". Chapter I provides the machinery for all the subsequent chapters.

The principal part of the paper (Chapters II and V) is devoted to internal characterizations of the $\mathscr{L}_{\infty}$ - and $\mathscr{L}_{1}$-spaces of Lindenstrauss and Pełczyński. These characterizations are in terms of the behavior of various classes of operators from or into these spaces. As a by-product an apparently new characterization of Hilbert spaces is obtained.

Finally, Chapter VI is a summary of the known characterizations of $\mathscr{L}_{1}$ - and $\mathscr{L}_{\infty}$-spaces.
\end{abstract}

Introduction. The notation used will be that of [29] and [35] where one can find the basic theorems of topological vector spaces that we shall use. We shall be considering only normed spaces, and for the most part Banach spaces.

This work is based on the Memoir of Grothendieck [7] (see also [8], [9], [30], [23]) and the papers [18], [19] of Lindenstrauss, Pełczyński and Rosenthal. No attempt will be made to give proofs, or even detailed statements of the theorems of [7] that we shall be using. We shall list below the theorems basic to this work.

The word operator (and sometimes map) will mean a bounded linear transformation. We shall denote by $\mathscr{L}(E, F)$ the operators from $E$ to $F$. By an isomorphism, we shall mean a one-to-one operator that is open. We shall usually say whether the isomorphism is onto or not; isometry has the usual meaning. If

Received by the editors May 26, 1970.

AMS 1970 subject classifications. Primary 46B99, 47B10; Secondary 46M05.

Key words and phrases. Absolutely summing operators, nuclear operator, fully nuclear operator, completely nuclear operator, $\mathscr{L}_{p}$-spaces, sufficiently Euclidean spaces.

(1) Portions of this paper appear in the dissertation of the first named author prepared under the direction of the second at Louisiana State University.

$\left.{ }^{2}\right)$ Supported by NSF-GP-11761. 
$T: E \rightarrow F$ is an operator, the range of $T$ is $F$ and the image of $T$ is the subset $T(E)$ of $F$. A projection $P$ is an operator in $\mathscr{L}(E, E)$ such that $P^{2}=P$. If $T: E \rightarrow F$ is an operator and $E_{0} \subseteq E$ we shall denote by $\left.T\right|_{E_{0}}$ the restriction of $T$ to $E_{0}$.

If $\left\{x_{\alpha}\right\} \subseteq E$ where $E$ is a Banach space, then by $\left[x_{\alpha}\right]$ we mean the closed, linear span of $\left\{x_{\alpha}\right\}$ in $E$; that is, the smallest closed subspace of $E$ containing $\left\{x_{\alpha}\right\}$.

The $\mathscr{L}_{p}$-spaces. Here we define the $\mathscr{L}_{p}$-spaces of [18]. We will only concern ourselves in this work with the cases $p=1$ or $\infty$.

Definition 0.1. If $E$ and $F$ are isomorphic Banach spaces, the distance coefficient of $E$ and $F, d(E, F)$, is defined by

$$
d(E, F)=\inf \left\{\|T\| \cdot\left\|T^{-1}\right\|: T: E \rightarrow F, T \text { is an onto isomorphism }\right\} .
$$

We shall denote by $c_{0}, l_{p}, 1 \leqq p \leqq \infty$, the usual sequence spaces with their standard norm. By $l_{p}^{n}, 1 \leqq p \leqq \infty, n=1,2,3, \ldots$, we shall mean the $n$-fold product of the complex (or real) numbers with the following norm:

For $1 \leqq p<\infty$,

$$
\left\|\left(t_{1}, \ldots, t_{n}\right)\right\|=\left(\sum_{i=1}^{n}\left|t_{i}\right|^{p}\right)^{1 / p}
$$

For $p=\infty$,

$$
\left\|\left(t_{1}, \ldots, t_{n}\right)\right\|=\max _{1 \leqq i \leqq n}\left|t_{i}\right| .
$$

As usual, we shall denote by $L_{p}(S, \mu), 1 \leqq p \leqq \infty$, the space of $p$-integrable functions given by a measure $\mu$ (in the sense of Bourbaki) on a locally compact Hausdorff space $S$ (actually equivalence classes of such functions!).

Let $\lambda \geqq 1$ be given and $1 \leqq p \leqq \infty$. A space $E$ is an $\mathscr{L}_{p, \lambda}$-space if for each finitedimensional $F \subseteq E$ there exists a finite-dimensional subspace $B$ with $F \subseteq B \subseteq E$ such that $d\left(B, l_{p}^{n}\right) \leqq \lambda$ where $n=\operatorname{dim}(B)$, the dimension of $B$.

A space $E$ is an $\mathscr{L}_{p}$-space if $E$ is an $\mathscr{L}_{p, \lambda}$-space for some $\lambda \geqq 1$. We shall frequently use the result that the dual of an $\mathscr{L}_{p}$-space is an $\mathscr{L}_{q}$-space, $1 \leqq p \leqq \infty$ and $1 / p+1 / q=1$ [18], [19].

Injective spaces.

Definition 0.2. The projection constant $P(E)$ of a Banach space $E$ is defined by

$$
P(E)=\operatorname{Sup} \inf \{\|P\|: P: F \rightarrow E \text { a projection }\}
$$

where the supremum is taken over all spaces having a subspace linearly isometric to $E$.

A space $E$ with $P(E)<+\infty$ is called injective ( $P$-space, $P_{\lambda}$-space for some $\lambda \geqq 1$ ). If $\operatorname{dim} E \leqq n$ then $E$ is injective and $P(E) \leqq n$ [28].

We will repeatedly use the result of Lindenstrauss and Rosenthal [19] that the dual of an $\mathscr{L}_{1}$-space is injective and the following result of Goodner [36].

THEOREM 0.3. The following are equivalent:

(a) E is injective; 
(b) for any pair of Banach spaces $Y, Z, Y \supset E$ and any $T \in \mathscr{L}(E, Z)$ there is an extension $\tilde{T}: Y \rightarrow Z$ (with $\|\tilde{T}\| \leqq \lambda\|T\|$ if $E$ is a $P_{\lambda^{-}}$-space).

Series and bases. A series $\sum_{i=1}^{\infty} x_{i}$ in a Banach space $E$ is said to converge absolutely if $\sum_{i=1}^{\infty}\left\|x_{i}\right\|<+\infty$ and to converge unconditionally if $\sum_{i=1}^{\infty} \varepsilon_{i} x_{i}$ converges for all choices of (complex) numbers $\varepsilon_{i},\left|\varepsilon_{i}\right|=1$.

By a biorthogonal system $\left(x_{i}, f_{i}\right)$ in $E$ we mean sequences $\left(x_{i}\right) \subset E,\left(f_{i}\right) \subset E^{\prime}$ such that $f_{i}\left(x_{j}\right)=\delta_{i j}$. The expression $\sum_{i=1}^{\infty} f_{i}(x) x_{i}$ is the formal expansion of $x \in E$ with respect to the biorthogonal system $\left(x_{i}, f_{i}\right)$. A (Schauder) basis for $E$ is a biorthogonal system $\left(x_{i}, f_{i}\right)$ such that the formal expansion of each $x \in E$ converges to $x$ in the norm topology of $E$. An unconditional basis is a basis in which each expansion converges unconditionally.

The basis constant is the supremum of the norms of the partial sum operators.

We will have occasion to use the following trivial special case of a theorem of Day. (Indeed Day's book [35] is an excellent source for information about series and bases.)

THEOREM 0.4. Let $E$ be an infinite-dimensional normed space. Then there is a biorthogonal system $\left(x_{i}, f_{i}\right)$ in $E$ with $\left\|x_{i}\right\|=\left\|f_{i}\right\|=1$ for each $i$.

Tensor products. On the algebraic tensor product $E \otimes F$ of two Banach spaces $E$ and $F$ we shall consider only two topologies. The first, the $\pi$-topology (projective, greatest cross-norm-topology) $[7, \S 1$, no. 1] is defined as follows:

For $u \in E \otimes F$,

$$
\|u\|=\inf \left\{\sum_{i=1}^{n}\left\|x_{\imath}\right\|\left\|y_{i}\right\|: u=\sum_{i=1}^{n} x_{i} \otimes y_{i}\right\}
$$

where the inf is over all possible representations of $u$. We denote the completion of $E \otimes F$ with the $\pi$-topology by $E \hat{\otimes} F$.

We will repeatedly use the following result of Grothendieck [7, §2, no. 1] (for an elementary proof due to Pietsch see [29]).

THEOREM 0.5. An element $u \in E \hat{\otimes} F$ can be represented in the following form: $u=\sum_{i=1}^{\infty} \lambda_{i} x_{i} \otimes y_{i}$ where $\left(\lambda_{i}\right) \in l_{1}, x_{i} \rightarrow 0$ and $y_{i} \rightarrow 0$.

We also need the following result (also due to Grothendieck) which is easy to prove:

THEOREM 0.6. The dual of $E \hat{\otimes} F$ is the space of continuous bilinear forms on $E \times F$, which is the same as $\mathscr{L}\left(E, F^{\prime}\right)$ or $\mathscr{L}\left(F, E^{\prime}\right)$, with the duality given by the following: if $u=\sum_{i=1}^{n} x_{i} \otimes y_{i}$ is in $E \otimes F$ and $T$ is in $\mathscr{L}\left(E, F^{\prime}\right)$ then

$$
\langle u, T\rangle=\sum_{i=1}^{n}\left\langle T x_{i}, y_{i}\right\rangle \text {. }
$$

The second topology, the $\varepsilon$-topology (inductive, least cross-norm-topology) [7, $\S 3$, no. 1] on $E \otimes F$ is defined as follows: the $\varepsilon$-topology is the topology induced on 
$E \otimes F$ by considering it as a subspace of $\mathscr{L}\left(E^{\prime}, F\right)$ via the canonical map $e \otimes f \rightarrow T$ where $T\left(e^{\prime}\right)=\left\langle e^{\prime}, e\right\rangle f$. We shall denote the $\varepsilon$-completion of $E \otimes F$ by $E \otimes F$.

The dual of $E \ddot{\otimes} F$ has also been characterized by Grothendieck. The dual is a subset of the bilinear forms on $E \times F$ called the integral bilinear forms and denoted by $J(E, F)$.

If $E_{0} \subset E$ and $F_{0} \subset F$ there is an obvious operator $J: E_{0} \otimes F_{0} \rightarrow E \otimes F$. In the $\pi$-topology, the adjoint $J^{\prime}$ is the restriction of a bilinear form on $E \times F$ to a bilinear form on $E_{0} \times F_{0}$; and, in the $\varepsilon$-topology $J^{\prime}$ is onto (because, in the case, $J$ is always an isometry), and is thus the restriction of an integral bilinear form on $E \times F$ to an integral bilinear form on $E_{0} \times F_{0}$.

The (metric) approximation property.

Definition 0.7. A Banach space $E$ has the approximation property if for each compact set $K \subset E$ there is an operator $T \in \mathscr{L}(E, E)$ with finite-dimensional range such that $\|x-T x\| \leqq 1$ for $x \in K$. If $T$ can be chosen so that $\|T\| \leqq 1$ then $E$ is said to have the metric approximation property.

Of course [7] is the classic work on the approximation property. We need only the following results from [7, §5]: Define the operator $I: E \hat{\otimes} F \rightarrow J\left(E^{\prime}, F^{\prime}\right)$ as follows: if $u=\sum_{i=1}^{\infty} \lambda_{i} x_{i} \otimes y_{i} \in E \hat{\otimes} F$ and $v=\sum_{j=1}^{n} f_{j} \otimes g_{j} \in E^{\prime} \otimes F^{\prime}$ and $\|v\|_{\varepsilon} \leqq 1$, then

$$
|\langle v, I u\rangle|=\left|\sum_{i=1}^{\infty}\left(\sum_{j=1}^{n} \lambda_{i} f_{j}\left(x_{i}\right) g_{j}\left(y_{i}\right)\right)\right| \leqq \sum_{i=1}^{\infty}\left|\lambda_{i}\right|\left\|x_{i}\right\|\left\|y_{i}\right\|
$$

which shows that $I$ is a bounded linear operator.

Also since $\varepsilon \leqq \pi$ there is an obvious (canonical) map $J: E \hat{\otimes} F \rightarrow E ð F$.

THEOREM 0.8. The following are equivalent:

(a) E has the approximation property;

(b) for each Banach space $F$ the mapping $J$ above is one-to-one; and

(c) for each Banach space $F$ the mapping I defined above is one-to-one.

Concerning the metric approximation property we shall need the following [7, p. 181, Corollaire 1, \#2].

THEOREM 0.9. If E has the metric approximation property then the map I above is an into isometry for each Banach space $F$.

Certain classes of operators. In the remainder of this paper we will be primarily concerned with four (usually distinct) classes of operators.

Absolutely summing operators.

Definition 0.10. An operator $T \in \mathscr{L}(E, F)$ is absolutely summing if for each unconditionally convergent series $\sum_{i=1}^{\infty} x_{i}$ in $E$, the series $\sum_{i=1}^{\infty} T x_{i}$ is absolutely convergent in $F$. These operators were introduced by Grothendieck [7, p. 55]. He called these operators "semi-integral adroit". The term "absolutely summing" appears to be due to Pietsch [24], who first systematically studied these operators. We need a consequence of Pietsch's characterization [24] of these operators. 
THEOREM 0.11. Let $\Omega$ be a compact Hausdorff space, $W$ a closed subspace of $C(\Omega)$ and $T \in \mathscr{L}(W, F), F$ a Banach space. Then $T$ is absolutely summing if and only if there is a $\mu \in C(\Omega)^{\prime}, \mu \geqq 0$ such that $\|T x\| \leqq \mu(|x|)$, for all $x \in W$.

We let $\|T\|_{\text {as }}=\inf \{\mu(\Omega)\}$ where the infimum is over all $\mu$ satisfying 0.11 .

We denote the absolutely summing operators from $E$ to $F$ by $\operatorname{AS}(E, F)$.

It is immediate from 0.11 that any $T \in \operatorname{AS}(E, F)$ admits the following factorization:

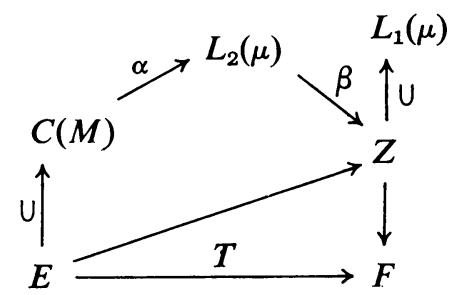

where $M$ is the unit ball of $E^{\prime}$ with its $w^{*}$-topology and $\subset$ denotes (canonical) inclusion and $\alpha, \beta$ are canonical. Here $Z$ denotes the closure of the image of $E$ under the indicated maps. We have given the factorization in a fashion somewhat different from that usually given (see e.g. [24]) since we need later that an absolutely summing operator $T$ factors through a subspace of $L_{1}(\mu)$ for some measure $\mu$.

Integral and nuclear operators. An operator $T \in \mathscr{L}(E, F)$ induces in a natural fashion a bilinear form $T_{B}$ on $E \times F^{\prime}$. Indeed, $T_{B}\left(e, f^{\prime}\right)=\left\langle T e, f^{\prime}\right\rangle$.

Definition 0.13. (i) An operator $T \in \mathscr{L}(E, F)$ is integral if the induced bilinear form $T_{B}$ is an integral bilinear form on $E \times F^{\prime}$.

(ii) An operator $T \in \mathscr{L}(E, F)$ is nuclear if there exists a $u \in E^{\prime} \hat{\otimes} F$ such that $J(u)=T$ where $J: E^{\prime} \hat{\otimes} F \rightarrow E^{\prime} \otimes F$ is the canonical operator. It is immediate from 0.5 that if $T$ is nuclear then there are sequences $\left(\lambda_{i}\right) \in l_{1},\left(f_{i}\right) \subset E^{\prime},\left(y_{i}\right) \subset F, f_{i} \rightarrow 0$, $y_{i} \rightarrow 0$, such that $T x=\sum_{i=1}^{\infty} \lambda_{i} f_{i}(x) y_{i}$ for $x \in E$. We let $\mathrm{I}(E, F)$ and $\mathrm{N}(E, F)$ denote respectively the integral and nuclear operators from $E$ to $F$. The nuclear norm of a nuclear operator $T$ is the infimum of the $\pi$-norms of the corresponding tensors.

We consistently use the following two results. The first is immediate from the above discussion and the second is a profound result of Grothendieck.

THEOREM 0.14. If $T \in \mathrm{N}(E, F)$ then $T$ admits the following factorization:

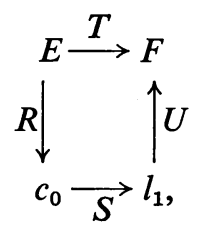

where $S \in \mathrm{N}\left(c_{0}, l_{1}\right)$. Indeed, if $T=\sum_{i=1}^{\infty} \lambda_{i} f_{i} \otimes y_{i}, f_{i} \rightarrow 0, y_{i} \rightarrow 0,\left(\lambda_{i}\right) \in l_{1}$, let $R(x)$ $=\left(\left\langle x, f_{i}\right\rangle\right), S\left(\xi_{i}\right)=\left(\lambda_{i} \xi_{i}\right)$ and $U\left(\eta_{i}\right)=\sum_{i=1}^{\infty} \eta_{i} y_{i}$. 
It is clear from the definition that a nuclear operator is compact. Grothendieck [7] has shown that the canonical injection from $L_{\infty}(\mu) \rightarrow L_{1}(\mu)$ is integral and so integral maps are not in general nuclear. The following result of Grothendieck [7, Lemma 14, p. 133] whose proof rests on a strong version of the Dunford-Pettis theorem is somewhat surprising.

THEOREM 0.15. If $T: E \rightarrow F$ is integral and $S: F \rightarrow G$ is weakly compact then $S T$ is nuclear. If $T: E \rightarrow F$ is weakly compact and $S: F \rightarrow G$ is integral and if either $E^{\prime \prime}$ or $G^{\prime}$ has the approximation property then $S T$ is nuclear [7, Theorem 10, pp. 132-133; Proposition 15, p. 86].

Quasi-nuclear operators. The quasi-nuclear operators which we now define were introduced by Pietsch [23], [25].

Definition 0.16. A $T \in \mathscr{L}(E, F)$ is quasi-nuclear if there is an absolutely convergent series $\sum_{i=1}^{\infty} f_{i}$ in $E^{\prime}$ such that $\|T x\| \leqq \sum_{i=1}^{\infty}\left|f_{i}(x)\right|$ for each $x \in E$. We denote by $\mathrm{QN}(E, F)$ the quasi-nuclear operators from $E$ to $F$.

The following is due to Pietsch.

Theorem 0.17. Let $T \in \mathrm{QN}(E, F)$ and $\Gamma$ an index set such that $F \subset l_{\infty}(\Gamma)$. Then if $\varphi$ is the injection of $F$ into $l_{\infty}(\Gamma), \varphi T \in \mathrm{N}\left(E, l_{\infty}(\Gamma)\right)$.

In Chapters II and IV we introduce the concepts of fully nuclear and completely nuclear operators.

In general the following situation occurs:

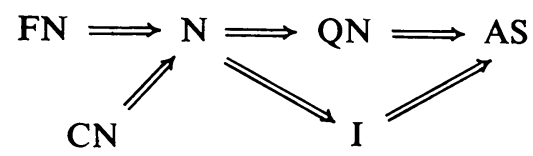

Extensions and liftings. We have briefly mentioned extensions of operators in the introductory section on injective spaces. It is known that the injective spaces are precisely the spaces having the "into" or "from" extension property for all operators. However, it is clear from the definition that

THEOREM 0.18. Nuclear operators enjoy the (nuclear) extension property.

If $\varphi: Z \rightarrow Y$ is an onto operator and $T \in \mathscr{L}(E, Y)$, an operator $\tilde{T}$ completing the triangle

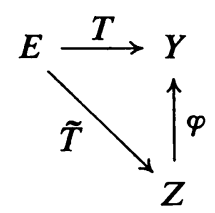

is called a lifting of $T$. It has been shown by Köthe [39] that the $l_{1}(\Gamma)$ spaces are precisely the spaces admitting liftings for all operators. Again from the definition it is clear that 
THEOREM 0.19. Every nuclear operator enjoys the (nuclear) lifting property.

Finally, we mention that some of the results of this paper have been announced in $[42, \mathrm{a}, \mathrm{b}]$.

The authors would like to express their appreciation to Professor Aldo Lazar for reading the manuscript and making many valuable suggestions.

\section{Sufficiently Euclidean Banach Spaces}

In this chapter we introduce a class of Banach spaces having finite-dimensional subspaces of a certain type. We make this precise below.

1. Sufficiently Euclidean Banach spaces. One of the most profound results in Banach space theory is the following theorem of A. Dvoretzky [4] concerning spherical sections of convex bodies in Banach spaces.

THEOREM I.1 (DVORETZKY). For each $\varepsilon>0$ and each positive integer $n$, there exists a positive integer $n(\varepsilon)$ such that if $E$ is any Banach space and the dimension of $E$ is greater than $n(\varepsilon)$, then there exists a subspace $F$ of $E$ such that $d\left(F, l_{2}^{n}\right) \leqq 1+\varepsilon$.

Thus, in each infinite-dimensional Banach space, there are finite-dimensional subspaces of arbitrarily large dimension, nearly isometric to Euclidean spaces. The property we need is in a certain sense stronger.

Definition I.2. A Banach space $E$ is sufficiently Euclidean if there is a positive constant $C$ and sequences of operators $\left\{J_{n}\right\},\left\{P_{n}\right\}$ such that

$$
l_{2}^{n} \stackrel{J_{n}}{\longrightarrow} E \stackrel{P_{n}}{\longrightarrow} l_{2}^{n}
$$

and $P_{n} J_{n}=I_{n}$, the identity operator on $l_{2}^{n}$, and $\left\|P_{n}\right\| \cdot\left\|J_{n}\right\| \leqq C$.

REMARK I.3. (i) Obviously we may assume $\left\|P_{n}\right\|=1$ and $1 \leqq\left\|J_{n}\right\| \leqq C$ for each $n$.

(ii) It is immediate that any space isomorphic to a Hilbert space (i.e., any $\mathscr{L}_{2}$ space) is sufficiently Euclidean.

(iii) If $F$ is sufficiently Euclidean and complemented in $E$, then $E$ is sufficiently Euclidean.

Remark I.3(iii) shows that the property of being sufficiently Euclidean actually tells you very little about the space. However, as a technical device, this notion seems to warrant study.

Let us compare Definition I.2 with Theorem I.1. While Dvoretzky's theorem says that in an infinite-dimensional Banach space there are many, nearly isometric copies of $l_{2}^{n}$ for arbitrarily large $n$, it says nothing about the norm of a projection onto one of these subspaces. For a space to be sufficiently Euclidean we are requiring that there be a sequence of finite-dimensional subspaces of increasing dimension such that each is "uniformly isomorphic" to $l_{2}^{n}$ for the appropriate $n$, and the projections onto these spaces are uniformly bounded.

From the definition, it is not immediately clear which Banach spaces are sufficiently Euclidean. It might appear that the only such spaces are those that 
isomorphically have Hilbert space as a complemented subspace. In fact, the class of sufficiently Euclidean spaces is rather large, and contains the classical reflexive spaces, as well as some hereditarily nonreflexive spaces.

Our first result is a consequence of Zippin's modification [34] of the "Principle of Local Reflexivity," the main result of [19]. This modification resulted from a question originally posed by the authors.

The principle of local reflexivity. Let $G$ be a finite-dimensional subspace of $E^{\prime \prime}$ and $P: E^{\prime \prime} \rightarrow G$ a projection. Then, for each $\varepsilon>0$ there is a subspace $G_{0}$ of $E$ and an isomorphism $S: G \rightarrow G_{0}$ such that

(i) $S$ restricted to $G \cap E$ is the identity;

(ii) $\|S\|\left\|S^{-1}\right\| \leqq 1+\varepsilon$; and

(iii) there is a projection $Q: E \rightarrow G_{0}$ such that $\|Q\| \leqq(1+\varepsilon)\|P\|$.

Property (iii) was added by M. Zippin. This theorem is one of the most remarkable results obtained in the past few years and appears to be a powerful tool. For some of its uses see [19]. We give one application now.

THEOREM I.4. A Banach space $E$ is sufficiently Euclidean if and only if $E^{\prime}$ is also sufficiently Euclidean.

Proof. If $E$ is sufficiently Euclidean, with $C$ the constant and $\left\{P_{n}\right\}$ and $\left\{J_{n}\right\}$ the given operators, then clearly the adjoints $\left\{P_{n}^{\prime}\right\},\left\{J_{n}^{\prime}\right\}$ and the same constant $C$ will suffice for $E^{\prime}$. The converse follows immediately from the principle of local reflexivity above. In fact, if $E^{\prime}$ is sufficiently Euclidean, then $E^{\prime \prime}$ is also, so we have the operators $\left\{J_{n}\right\},\left\{P_{n}\right\}$ and the constant $C$ given by the definition. Let $\varepsilon=1, J_{n} P_{n}$ be the projection, $J_{n} P_{n}\left(E^{\prime \prime}\right)$ the finite-dimensional subspace of $E^{\prime \prime}$, then we obtain an isomorphism $S_{n}: J_{n} P_{n}\left(E^{\prime \prime}\right) \rightarrow E$, and a projection $Q_{n}: E \rightarrow E$ such that $Q_{n}(E)$ $=S_{n} J_{n} P_{n}\left(E^{\prime \prime}\right)$ with $\left\|S_{n}\right\| \cdot\left\|S_{n}^{-1}\right\| \leqq 2$ and $\left\|Q_{n}\right\| \leqq 2 C$; thus $\left\{S_{n} P_{n}\right\}$ and $\left\{J_{n} S_{n}^{-1} Q_{n}\right\}$ are the desired operators and $4 C^{2}$ the desired constant.

THEOREM I.5. For $1<p<\infty, l_{p}$ is sufficiently Euclidean.

Proof. The following proof is essentially contained in [21]. Let $\gamma_{i}$ denote the $i$ th Rademacher function, that is $\gamma_{i}(t)=\operatorname{sgn} \sin \left(2^{i-1} \pi t\right)$, and consider $R_{n}=$ $\left[\gamma_{1}, \ldots, \gamma_{n}\right] \subset L_{p}[0,1]$. Let $G_{2^{n}} \subset L_{p}[0,1]$ be the space spanned by $\chi_{\left[(k-1) / 2^{n}, k / 2^{n}\right]}$, $k=1, \ldots, 2^{n}, n=1,2,3, \ldots$ By extending the map $e_{k} \rightarrow \chi_{\left[(k-1) / 2^{n}, k / 2^{n}\right]}$, where $e_{k}$ is the $k$ th unit vector of $l_{p}^{2^{n}}$, it is easy to see that the resulting operator $T_{n}: l_{p}^{2^{n}} \rightarrow G_{2^{n}}$ is an isometry. By the definition of $\gamma_{i}$ we have $R_{n} \subset G_{2^{n}}$ for each $n$ and, by the classical Khinchin inequality, $R_{n}$ is isomorphic to $l_{2}^{n}$ and the norm of the isomorphism, $C_{p}$, depends only on $p$. Let

$$
Q_{n}(x)=\sum_{i=1}^{n} \int_{0}^{1} x(t) \gamma_{i}(t) d t \gamma_{i} .
$$


Clearly $Q_{n}: L_{p}[0,1] \rightarrow R_{n}$, and by the orthogonality properties of the Rademacher system it follows that $Q_{n}^{2}=Q_{n}$ for each $n$, that is $Q_{n}$ is a projection of $L_{p}[0,1]$ onto $R_{n}$ for each $n$, and $\left\|Q_{n}\right\| \leqq M_{p}$, a constant depending only on $p$ [13, p. 245]. (This much of the proof shows that $L_{p}[0,1]$ is sufficiently Euclidean for $1<p<\infty$.) Let $S_{n}: G_{2^{n}} \rightarrow R_{n}$ be the restriction of $Q_{n}$ to $G_{2^{n}}$ and let $U_{n}: l_{p} \rightarrow l_{p}^{2^{n}}$ be the natural projection. Then $P_{n}=T_{n}^{-1} S_{n} T_{n} U_{n}$ is a projection of $l_{p}$ onto a subspace whose distance from $l_{2}^{n}$ is no more than $C_{p}$. Hence, we have that $l_{p}$ is sufficiently Euclidean.

From [18] it follows that each $\mathscr{L}_{p}$-space, $1 \leqq p<\infty$, has a complemented subspace isomorphic to $l_{p}$. Thus we have the following result.

COROllary I.6. For $1<p<\infty$, an $\mathscr{L}_{p}$-space is sufficiently Euclidean.

The cases $p=1$ or $p=\infty$ will be considered below.

It is well known [1] and [2], that for $p \neq 2, l_{p}$ contains no isomorphic copy of $l_{2}$.

Our next result is an easy consequence of the definition.

THEOREM I.7. A Banach space $E$ is sufficiently Euclidean if and only if each subspace of finite codimension in $E$ is sufficiently Euclidean.

Proof. It is immediate from Remark I.3(iii) that if $E$ has a sufficiently Euclidean subspace of finite codimension, then $E$ is sufficiently Euclidean. For the converse, let $F$ be a subspace of codimension $m, 1 \leqq m<\infty$. If we have the constant $C$ and the operators $J_{n}: l_{2}^{n} \rightarrow E, P_{n}: E \rightarrow l_{2}^{n}$ given by Definition I.2, we must have for $n>m$ that dimension $\left(F \cap J_{n}\left(l_{2}^{n}\right)\right)>n-m$. There is a projection $Q_{n}: l_{2}^{n} \rightarrow P_{n} J_{n}\left(l_{2}^{n}\right)$ such that $\left\|Q_{n}\right\|=1$. If we let $S_{n}$ be the restriction of $P_{n}$ to $F$, then $\left\{J_{n} Q_{n}\right\}$ and $\left\{S_{n} Q_{n}\right\}$, $n \geqq m+1$, are the desired operators, and the same constant $C$ will suffice.

Remark I.8. (i) It follows from Theorem I.7 that if $E=E^{0}$ is a quasi-reflexive space [3], $E^{n}=\left(E^{n-1}\right)^{\prime}$ and if there is a $K$ such that $E^{K}$ is sufficiently Euclidean then it follows (without recourse to local reflexivity) that $E^{n}$ is sufficiently Euclidean for each $n$.

(ii) Let us mention that for every positive integer $n$ there is a sufficiently Euclidean space which is quasi-reflexive of order $n$, namely the $n$-fold product of the space of James [12].

(iii) It is known that a Banach space $E$ such that each separable subspace of $E$ is isomorphic to Hilbert space is itself isomorphic to Hilbert space [17]. It is interesting to note that, for $1<p<\infty, l_{p}$ has the property that every subspace is sufficiently Euclidean. For, if $E$ is an infinite-dimensional subspace of $l_{p}$, then it contains an isomorphic copy of $l_{p}$, that is complemented in $l_{p}$, hence in $E$ [21]. Thus $E$ is sufficiently Euclidean.

We will return to properties of sufficiently Euclidean Banach spaces but first we introduce a wider class of spaces.

2. The two series property. We now introduce a property enjoyed by all sufficiently Euclidean Banach spaces. 
Definition I.9. A Banach space $E$ is said to have the two series property provided there exists unconditionally convergent series $\sum_{i=1}^{\infty} f_{i}$ in $E^{\prime}$ and $\sum_{i=1}^{\infty} x_{i}$ in $E$ such that

$$
\lim _{n \rightarrow \infty} \sum_{i=1}^{n}\left(\sum_{j=1}^{\infty} \frac{\left|f_{j}\left(x_{i}\right)\right|^{2}}{\left\|f_{j}\right\|}\right)^{1 / 2}=+\infty
$$

We abbreviate this by saying $E$ has Property (S).

Following [18] we say that a pair of Banach spaces $\langle E, F\rangle$ is unconditionally trivial if each $T \in \mathscr{L}(E, F)$ is absolutely summing. In [18] the exceedingly nontrivial result that if $E$ is an $\mathscr{L}_{1}$-space and $F$ an $\mathscr{L}_{2}$-space then $\langle E, F\rangle$ is unconditionally trivial is proved. This result is a generalization of an earlier result of Grothendieck and indeed follows readily from the "Grothendieck inequality" [18], [8].

We see the importance of spaces with Property (S) in the following theorem.

Theorem I.10. Let E be a Banach space with Property (S), and let $F$ be any Banach space. Then $\langle E, F\rangle$ is not unconditionally trivial.

Proof. It follows from [4] and [18] that if $\mathscr{L}(E, F)=\mathrm{AS}(E, F)$ then $\mathscr{L}\left(E, l_{2}\right)$ $=\mathrm{AS}\left(E, l_{2}\right)$. We shall prove there is an operator $T: E \rightarrow l_{2}$ that is not absolutely summing. Let $\sum_{i=1}^{\infty} f_{i}, \sum_{i=1}^{\infty} x_{i}$ be the series which exist by Definition I.9. Let $\left\{e_{j}\right\}$ be the usual orthonormal basis of $l_{2}$. Define $T: E \rightarrow l_{2}$ by $T(x)=\sum_{j=1}^{\infty}\left\|f_{j}\right\|^{-1 / 2} f_{j}(x) e_{j}$. If $C=\sup _{\|x\|=1} \sum_{j=1}^{\infty}\left|f_{j}(x)\right|$, which is finite because $\sum_{j=1}^{\infty} f_{j}$ is unconditionally convergent, then for $\|x\| \leqq 1$,

$$
\begin{aligned}
\|T x\| & =\left\|\sum_{j=1}^{\infty} \frac{f_{j}(x)}{\left\|f_{j}\right\|^{1 / 2}} e_{j}\right\|=\left(\sum_{j=1}^{\infty} \frac{\left|f_{j}(x)\right|^{2}}{\left\|f_{j}\right\|}\right)^{1 / 2} \\
& \leqq\left(\sum_{j=1}^{\infty}\left|f_{j}(x)\right|\right)^{1 / 2} \leqq C^{1 / 2} .
\end{aligned}
$$

Thus $T$ is a bounded operator. Also, we have

$$
\lim _{n \rightarrow \infty} \sum_{i=1}^{n}\left\|T x_{i}\right\|=\lim _{n \rightarrow \infty} \sum_{i=1}^{n}\left(\sum_{j=1}^{\infty} \frac{\left|f_{j}\left(x_{i}\right)\right|^{2}}{\left\|f_{j}\right\|}\right)^{1 / 2}=\infty
$$

That is, $T$ is not absolutely summing because the image $\left\{T x_{i}\right\}$ of the unconditionally convergent series $\left\{x_{i}\right\}$ is not absolutely convergent.

We now determine the relationship between sufficiently Euclidean Banach spaces and those with Property (S).

THEOREM I.11. A sufficiently Euclidean Banach space E has Property (S).

Proof. Choose $\left\{t_{k}\right\}$ in $l_{4}$ but not in $l_{3}$ and $t_{k}>0$. Block $\left\{t_{k}\right\}$ such that

$$
\sum_{j=1}^{\infty}\left(\sum_{k=n_{j}+1}^{n_{j+1}} t_{k}^{4}\right)^{1 / 2}=L<\infty,
$$


and $0=n_{1}<n_{2}<\cdots, n_{j} \rightarrow \infty$ as $j \rightarrow \infty$. Let $m_{j}=n_{j+1}-n_{j}$; if $E$ is sufficiently Euclidean we must have operators $\left\{J_{j}\right\},\left\{P_{j}\right\}$, and a constant $C>0$ such that

$$
l_{2}^{m_{j}} \stackrel{J_{j}}{\longrightarrow} E \stackrel{P_{j}}{\longrightarrow} l_{2}^{m_{j}}
$$

where $P_{j} J_{j}$ is the identity on $l_{2}^{m_{j}}$ and $\left\|P_{j}\right\| \cdot\left\|J_{j}\right\| \leqq C$. If $\left\{e_{j i}\right\}$ denotes the usual basis of $l_{2}^{m}$ then it follows as in [4, Main Lemma] that if $J_{j}\left(e_{j i}\right)=x_{n_{j}+i}$ then $\sum_{i=1}^{\infty} t_{i}^{2} x_{i}$ is unconditionally convergent, as is $\sum_{i=1}^{\infty} t_{i}^{2} f_{i}$ where $f_{n_{j}+i}=P_{j}^{\prime}\left(e_{j i}\right)$, and we may assume $\left\|P_{j}\right\|=1,\left\|J_{j}\right\| \leqq C$. Here, of course, $\left(x_{i}\right)$ and $\left(f_{i}\right)$ are ordered by the corresponding blocks. Thus,

$$
\sum_{i=1}^{n}\left(\sum_{j=1}^{n} \frac{t_{i}^{4} t_{j}^{4}\left|f_{j}\left(x_{i}\right)\right|^{2}}{\left|t_{j}\right|^{2}\left\|f_{j}\right\|}\right)^{1 / 2} \geqq \sum_{i=1}^{n} \frac{t_{i}^{3}\left|f_{i}\left(x_{i}\right)\right|}{\left\|f_{i}\right\|} \geqq \sum_{i=1}^{n} t_{i}^{3} .
$$

Since $\lim _{n} \sum_{i=1}^{n} t_{i}^{3}=\infty$, we also have

$$
\lim _{n \rightarrow \infty} \sum_{i=1}^{n}\left(\sum_{j=1}^{\infty} \frac{\left|f_{j}\left(x_{i}\right)\right|^{2}}{\left\|f_{j}\right\|}\right)^{1 / 2}=\infty .
$$

Thus $E$ has Property (S).

There are several corollaries to Theorems I.10 and I.11, and other applications will be given in Chapter II.

CoRollaRY I.12. No $\mathscr{L}_{1}$ - or $\mathscr{L}_{\infty}$-space is sufficiently Euclidean.

Proof. By the profound result mentioned above an operator $T$ from an $\mathscr{L}_{1}$-space to an $\mathscr{L}_{2}$-space is absolutely summing. By Theorem I.10, an $\mathscr{L}_{1}$-space does not have Property (S) hence is not sufficiently Euclidean. Since $\mathscr{L}_{1}$ - and $\mathscr{L}_{\infty}$-spaces are in duality [19], Theorem I.4 says that $\mathscr{L}_{\infty}$-spaces are not sufficiently Euclidean.

REMARK I.13. If $E$ contains an isomorphic copy of $c_{0}$, or $l_{p}, p>2$, then $E$ has Property (S). (Hence, most, and perhaps all, $\mathscr{L}_{\infty}$-spaces have Property (S) [19].)

Proof. Suppose $J: c_{0} \rightarrow E$ is such that $m\|x\| \leqq\|J x\| \leqq M\|x\|$. If we choose $f_{i}$ in $E^{\prime},\left\|f_{i}\right\| \leqq C, f_{i}\left(J e_{i}\right)=\delta_{i j}$ where $\left\{e_{j}\right\}$ is the usual basis of $c_{0}$, then certainly

$$
\sum_{j=1}^{\infty}\left(\frac{1}{j}\right)^{1 / 3} J e_{j} \text { and } \sum_{j=1}^{\infty}\left(\frac{1}{j}\right)^{4 / 3} f_{j}
$$

are unconditionally convergent (the latter is even absolutely convergent). And we have the following:

$$
\begin{aligned}
\lim _{n \rightarrow \infty} \sum_{i=1}^{n}\left(\sum_{j=1}^{\infty} \frac{\left|f_{j}\left(J e_{i}\right)\right|(1 / i)^{2 / 3}(1 / j)^{8 / 3}}{(1 / j)^{4 / 3}\left\|f_{j}\right\|}\right)^{1 / 2} & \geqq \lim _{n \rightarrow \infty} \sum_{i=1}^{n} \frac{1 / i}{\left\|f_{i}\right\|} \\
& \geqq \lim _{n \rightarrow \infty} \frac{1}{C} \sum_{i=1}^{n} \frac{1}{i}=\infty
\end{aligned}
$$

Thus $E$ has Property (S). The proof for $l_{p}, p>2$, is analogous. 
REMARK I.14. In Corollary I.12 we prove that an $\mathscr{L}_{\infty}$-space is not sufficiently Euclidean by using the results of Grothendieck, Pełczyński-Lindenstrauss, and Lindenstrauss-Rosenthal ([8], [18] and [19]) showing that an $\mathscr{L}_{1}$-space is not sufficiently Euclidean. It is interesting that another proof can be given avoiding use of the inequality of Grothendieck [8], [18] which is the main tool in the proof of the theorem that all operators from an $\mathscr{L}_{1}$-space to an $\mathscr{L}_{2}$-space are absolutely summing. It is known that a Banach space $E$ is an $\mathscr{L}_{\infty}$-space if and only if $E^{\prime \prime}$ is a $P_{\lambda}$-space for some $\lambda \geqq 1$ [19], [15]. Then by [15, Corollary 3, p. 16] if $B$ is a finitedimensional subspace of $\mathscr{L}_{\infty}$ on which there is a projection of norm no more than $M$, then $B$ is a $P_{\lambda M}$-space. If $E$ were sufficiently Euclidean, $C$ the constant and $\left\{E_{n}\right\}$ the sequence of subspaces given by Definition I.2, then by [28, Lemma 1, p. 246]

$$
(1 / C) P\left(l_{2}^{n}\right) \leqq P\left(E_{n}\right) \leqq C P\left(l_{2}^{n}\right)
$$

where $P\left(E_{n}\right)$ denotes the projection constant of $E_{n}$ [10]. By the above, $E_{n}$ is a

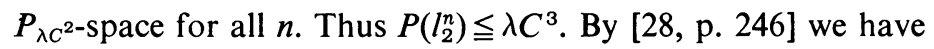

$$
P\left(l_{2}^{n}\right)=n \Gamma\left(\frac{1}{2} n\right) / \pi^{1 / 2} \Gamma\left(\frac{1}{2} n+\frac{1}{2}\right) .
$$

By Stirling's formula it easily follows that $P\left(l_{2}^{n}\right) \rightarrow \infty$. Thus $E$ is not sufficiently Euclidean. Again applying [19] and Theorem I.4 we obtain that an $\mathscr{L}_{1}$-space is not sufficiently Euclidean.

Although there are nonsufficiently Euclidean spaces, we do have the following result.

THEOREM I.15. Every infinite-dimensional Banach space E contains a sufficiently Euclidean subspace F. Moreover, F can be chosen to have a Schauder basis.

Proof. This theorem is essentially (of course, using different language) the result of Gurarii [11] concerning basic sequences in Banach spaces. We outline the proof. If $F$ and $G$ are subspaces of a Banach space $E$ then the inclination of $F$ and $G$, $\mathrm{I}(F, G)$, is defined by

$$
\mathrm{I}(F, G)=\inf \{\|x+y\|: x \in F,\|x\|=1, y \in G\} .
$$

If $\left\{x_{i}\right\}$ is a sequence in $E$ the index $\theta\left\{x_{i}\right\}$ of $\left\{x_{i}\right\}$ is defined by

$$
\theta\left\{x_{i}\right\}=\inf _{n}\left\{d\left(S_{n},\left[x_{i}: i>n\right]\right)\right\}
$$

where $S_{n}$ denotes the unit sphere of $\left[x_{i}: i \leqq n\right]$. It is well known [6] that a sequence is basic $\left\{x_{i}\right\}$ if and only if $\theta\left\{x_{i}\right\}>0$. To prove Theorem I.13 we state the following three lemmas.

Lemma I.16. Given $\varepsilon>0$, and a finite-dimensional subspace $F$ there is an infinitedimensional subspace $G$ of $E$ such that $\mathrm{I}(F, G)>1-\varepsilon$. 
Lemma I.17. Suppose the subspaces $F_{1}, F_{2}=G_{1} \oplus G_{2}$ and $F_{3}$ satisfy

(i) I $\left(G_{1}, G_{2}\right) \geqq \alpha>0$,

(ii) I $\left(F_{1} \oplus F_{2}, F_{3}\right) \geqq \beta>0$,

(iii) $\mathrm{I}\left(F_{1}, F_{2} \oplus F_{3}\right) \geqq \beta>0$,

then $\mathrm{I}\left(F_{1} \oplus G_{1}, G_{2} \oplus F_{3}\right) \geqq \alpha \beta^{2} /(2+\alpha)$.

LemMA I.18. Let $\left\{x_{i}^{1}, x_{i}^{2}, \ldots, x_{i}^{k_{i}}\right\}$ be bases of $k_{i}$-dimensional subspaces $P_{i} \subset E$ for which $\theta\left(x_{i}^{j}\right) \geqq \alpha>0$ for $j=1, \ldots, k_{i}, i=1,2, \ldots$, and for any integers $m, n$, with $m<n, \mathrm{I}\left(P_{1} \oplus \cdots \oplus P_{m}, P_{m+1} \oplus \cdots \oplus P_{n}\right) \geqq \beta>0$. Then $\left\{x_{i}^{j}\right\}$ is a basis for

$$
\left[x_{i}^{j}: j=1, \ldots, k_{i}, i=1,2, \ldots\right]
$$

and the index of this sequence is $\geqq \alpha \beta^{2} /(2+\alpha)$.

The proofs of Lemmas I.16, I.17 and I.18 can be derived from [11].

Now by using Theorem I.1 we apply the above with $\left\{x_{i}^{1}, \ldots, x_{i}^{k_{i}}\right\}$ corresponding under an $\varepsilon$-isometry to the unit vector basis of $l_{2}^{k_{i}}$. If $F=\left[x_{i}^{j}\right]$ then $F$ is clearly sufficiently Euclidean. The projections from $F$ onto $\left[x_{i}^{1}, \ldots, x_{i}^{k_{i}}\right]$ have norm no greater than $2 K$, where $K$ is the basis constant. This proves Theorem I.15.

CoROllaRy I.19. Every $\mathscr{L}_{1}-\left(\mathscr{L}_{\infty}\right)$-space contains a subspace with a basis that is not an $\mathscr{L}_{1}-\left(\mathscr{L}_{\infty}\right)$-space.

Proof. This follows immediately from Corollary I.12 and Theorem I.15.

This generalizes the examples given in [21], [16], for subspaces of $c_{0}$ and $l_{1}$ respectively.

Corollary I.19 can also be obtained from the results of [18].

COROLlaRY I.20. There exists hereditarily nonreflexive sufficiently Euclidean spaces.

Proof. This is immediate from the well-known result that $c_{0}$ and $l_{1}$ have no reflexive subspaces.

We restate in somewhat different, and more useful forms, some of the results we have obtained in this chapter.

THEOREM I.21. Let $E$ and $F$ be infinite-dimensional Banach spaces. Then there is an infinite-dimensional subspace $E_{0}$ of $E$ and an operator $T: E_{0} \rightarrow F$ that is not absolutely summing.

Proof. We have only to choose $E_{0}$ to be sufficiently Euclidean and apply Theorem I.10.

THEOREM I.22. Let $E$ and $F$ be infinite dimensional Banach spaces. Then there is an infinite-dimensional subspace $F_{0}$ of $F$ and an operator $T: E \rightarrow F_{0}$ such that $T^{\prime}$ is not absolutely summing (hence $T$ cannot be integral). 
Proof. As in Theorem I.11, we choose a sequence $\left\{t_{k}\right\}$ in $l_{4}$ but not $l_{3}$ and block (and re-index) such that $\sum_{i=1}^{\infty}\left(\sum_{j=1}^{k_{i}} t_{i j}^{4}\right)^{1 / 4}<\infty$. As in Theorem I.15, choose a sufficiently Euclidean space $F_{0}=\left[y_{i}^{j}\right]$, with corresponding basis constant $C / 2$ and the "blocks" $\left\{y_{i}^{1}, \ldots, y_{i}^{k_{i}}\right\}$ corresponding to the above blocking. By Theorem I.1 find $\varepsilon$-isometric copies of $l_{2}^{k_{i}}$, say $\left[f_{i}^{j}\right], j=1, \ldots, k_{i}, j=1,2, \ldots$, in $E^{\prime}$. Consider the operator

$$
T(x)=\sum_{i=1}^{\infty} \sum_{j=1}^{k_{i}} t_{i j} f_{i}^{j}(x) y_{i}^{j} .
$$

For $\|x\| \leqq 1$, we have

$$
\begin{aligned}
\|T x\| & =\left\|\sum_{i=1}^{\infty} \sum_{j=1}^{k_{i}} t_{i j} f_{i}^{j}(x) y_{i}^{j}\right\| \leqq \sum_{i=1}^{\infty}\left\|\sum_{j=1}^{k_{i}} t_{i j} f_{i}^{j}(x) y_{i}^{j}\right\| \\
& \leqq C(1+\varepsilon) \sum_{i=1}^{\infty}\left(\sum_{j=1}^{k_{i}} t_{i j}^{2}\left|f_{i}^{j}(x)\right|^{2}\right)^{1 / 2} \\
& \leqq C(1+\varepsilon)^{3 / 2} \sum_{i=1}^{\infty}\left(\sum_{j=1}^{k_{i}} t_{i j}^{2}\left|f_{i}^{j}(x)\right|\right)^{1 / 2} \\
& \leqq C(1+\varepsilon)^{3 / 2} \sum_{i=1}^{\infty}\left(\sup _{\|x\|=1} \sum_{j=1}^{k_{i}}\left|t_{i j}^{2} f_{i}^{j}(x)\right|\right)^{1 / 2} \\
& \leqq C(1+\varepsilon)^{3 / 2} \sum_{i=1}^{\infty}\left(\sup _{\left|\varepsilon_{j}\right|=1}\left\|\sum_{j=1}^{k_{i}} t_{i j}^{2} \varepsilon_{j} f_{i}^{j}\right\|\right)^{1 / 2} \\
& \leqq C(1+\varepsilon)^{2} \sum_{i=1}^{\infty}\left(\sum_{j=1}^{k_{i}} t_{i j}^{4}\right)^{1 / 4} \cdot
\end{aligned}
$$

Thus $T$ is a bounded linear operator. Proceeding exactly as in Theorem I.10 we see that $T^{\prime}: F_{0}^{\prime} \rightarrow E^{\prime}$ is not absolutely summing.

3. Questions. There are many problems concerning sufficiently Euclidean spaces which are unsolved. Of course, we have developed only a part of the theory of such spaces in order to apply it to the later chapters. Indeed as remarked earlier, the sufficiently Euclidean spaces appear to be useful primarily as a technical device. It appears to us that for this reason, if no other, the sufficiently Euclidean spaces warrant study as an entitity in themselves. Answers to the following questions would go a long way in furthering the theory and applications of these spaces.

(Q1) Is every reflexive space sufficiently Euclidean?

(Q2) If $E_{0}$ is a sufficiently Euclidean subspace of a conjugate Banach space $F^{\prime}$, is the $\sigma\left(F^{\prime}, F\right)$-closure of $E_{0}$ also sufficiently Euclidean $\left({ }^{3}\right)$ ?

We suspect that $(\mathrm{Q} 1)$ has an affirmative answer and that $(\mathrm{Q} 2)$ has a negative response.

$\left({ }^{3}\right)$ H. Rosenthal asserts that counterexamples to (Q2) can be found using methods of harmonic analysis. 
If, however, the response to (Q2) is affirmative, one could prove I.4 without recourse to the principle of local reflexivity. Also, an affirmative response to (Q2) would answer (Q8) in Chapter III affirmatively.

We conjecture, however, that there is a $\sigma\left(l_{1}, c_{0}\right)$-dense sufficiently Euclidean subspace of $l^{1}$.

Since the pair $\langle E, F\rangle$ unconditionally trivial implies that the pair $\left\langle E, l_{2}\right\rangle$ is unconditionally trivial it is natural, in view of I.10, to raise the following question.

(Q3) If $\left\langle E, l_{2}\right\rangle$ is not unconditionally trivial, does $E$ possess the two series property?

There is reason to believe that $(\mathrm{Q} 3)$ has an affirmative answer. Indeed, suppose there is an unconditionally convergent series $\sum x_{i}$ in $E$ and $T \in \mathscr{L}\left(E, l_{2}\right)$ such that $\sum\left\|T x_{i}\right\|=+\infty$. Let $f_{i}=T^{\prime}\left(T x_{i}\right) \in E^{\prime}$. Since $\sum T x_{i}$ is unconditionally convergent, so is $\sum f_{i}$. Since we can suppose without loss of generality that $f_{i} \neq 0$ for each $i$, we have

where $M=\|T\|^{-1 / 2}$.

$$
\begin{aligned}
\sum_{j}\left(\sum_{i} \frac{f_{i}\left(x_{j}\right)^{2}}{\left\|f_{i}\right\|}\right)^{1 / 2} & =\sum_{j}\left(\sum_{i} \frac{\left|\left\langle T x_{i}, T x_{j}\right\rangle\right|^{2}}{\left\|T^{\prime} T x_{i}\right\|}\right)^{1 / 2} \\
& \geqq \sum_{i}\left\|T^{\prime} T x_{i}\right\|^{-1 / 2}\left\|T x_{i}\right\|^{2} \geqq M \sum_{i}\left\|T x_{i}\right\|^{3 / 2},
\end{aligned}
$$

This crude estimate gives rise to the following discussion. Generalizing the notion of a $p$-absolutely summing operator, Pełczyński and Mitiagin have called a $T \in \mathscr{L}(E, F)(p, q)$-absolutely summing, $1 \leqq p, q<+\infty$, provided there is a constant $C$ such that for every finite subset $\left\{x_{1}, \ldots, x_{n}\right\}$ of $E$

$$
\left(\sum_{i=1}^{n}\left\|T x_{i}\right\|^{p}\right)^{1 / p} \leqq C \sup \left\{\left(\sum_{i=1}^{n}\left|x^{\prime}\left(x_{i}\right)\right|^{q}\right)^{1 / q}:\left\|x^{\prime}\right\|=1\right\}
$$

(see [40] for a discussion of $(p, q)$-absolutely summing operators in $l_{p}$-spaces).

(Q4) If $\left\langle E, l_{2}\right\rangle$ is not unconditionally trivial, is there a non- $(3 / 2,1)$-absolutely summing operator from $E$ to $l_{2}$ ?

It is obvious how (Q4) relates to the above. Also, (Q4) is similar to Problem 3, p. 319 of [18]. Moreover, (Q4) is related to the questions concerning the parameters $\alpha(E)$ and $\beta(E)$ of [17] and to a classical theorem of Orlicz.

\section{Fully Nuclear Operators}

1. The Grothendieck conjecture. Of the many conjectures made by A. Grothendieck in his classical memoir [7] we find the following to be one of the most interesting:

(C) If $E$ and $F$ are Banach spaces and $\mathscr{L}(E, F)=\mathrm{N}(E, F)$, then $E$ or $F$ must be finite dimensional.

In a sense, the nuclear operators between two Banach spaces are the only "constructible" operators, that is, the operators of the most elementary form that include the finite-dimensional operators.

To our knowledge this conjecture has only been considered in [7] and in the very important paper of Lindenstrauss and Pełczyński [18]. 
While we are not able to solve (C) in the generality asserted by Grothendieck we are able to give an affirmative answer to (C) under a somewhat more restricted hypothesis, which, however, does seem to include all the known cases.

We now define the concept of a fully nuclear operator.

Definition II.1. A bounded operator $T: E \rightarrow F, E$ and $F$ Banach spaces, is said to be fully nucelar if the astriction $T_{a}: E \rightarrow T(E)$ is nuclear.

Notation. FN $(E, F)$ will denote the fully nuclear operators from $E$ to $F$.

REMARK II.2. (i) Grothendieck [7, p. 40] has given a general method for obtaining nuclear operators that are not fully nuclear (using, of course, different terminology); this construction is possible because of the following theorem due to Grothendieck [7, p. 40]:

Let $F$ be a Banach space and $E$ a closed subspace of $F$ such that $E$ is complemented in $E^{\prime \prime}$. Then the canonical operator $J: E \hat{\otimes} E^{\prime} \rightarrow F \hat{\otimes} E^{\prime}$ is an into isomorphism if and only if $E$ is complemented in $F$.

The proof is immediate from the fact that $J$ is an isomorphism if and only if $J^{\prime}: \mathscr{L}\left(F, E^{\prime \prime}\right) \rightarrow \mathscr{L}\left(E, E^{\prime \prime}\right)\left(J^{\prime}\right.$ is just restriction) is onto which is true if and only if $E^{\prime \prime}$ is complemented in $F^{\prime \prime}$. Thus examples of nuclear, nonfully nuclear operators may be given by choosing a Banach space $F$ with the approximation property, a closed, noncomplemented, reflexive subspace $E$ with the approximation property. Then there exists an element $T$ of $F \hat{\otimes} E^{\prime}$ that is in the closure of $E \hat{\otimes} E^{\prime}$ but not in $E \hat{\otimes} E^{\prime}$. Regarding $T$ as an operator, $T: E \rightarrow F$, then $T(E) \subset E, T$ is nuclear, but the astriction $T_{a}: E \rightarrow F$ is not nuclear. Numerous examples are given later.

(ii) It is easily seen $[23$, p. 50$]$ that if $F_{0}$ is a dense subspace of $F, T: E \rightarrow F$, $T(E) \subseteq F_{0}, T \in \mathrm{N}(E, F)$, then $T$ is nuclear with respect to $F_{0}$. Thus in the definition of a fully nuclear operator, it does not matter whether we consider the astriction to the image or to the closure of the image.

(iii) Many of the difficulties in the theory of nuclear operators arise because the representation of a nuclear operator $T: E \rightarrow F$ depends on the range $F$ and not the image $T(E)$. Obviously, these difficulties are not present when we consider fully nuclear operators.

(iv) Remark (i) shows that there are nuclear, nonfully nuclear operators; we shall see later that in a certain sense each nuclear operator can be obtained from a fully nuclear operator.

We can now give the main result of this chapter.

TheOREM II.3. Let $E$ and $F$ be Banach spaces and suppose $\mathscr{L}(E, F)=\mathrm{FN}(E, F)$. Then $E$ or $F$ is finite dimensional. The converse is also true.

Proof. Suppose both $E$ and $F$ are infinite dimensional. By Theorem I.15 there is a sufficiently Euclidean subspace $F_{0}$ of $F$ and hence, by Theorem I.4, $F_{0}^{\prime}$ is sufficiently Euclidean. By Theorem I.22 there is an operator $T: E \rightarrow F_{0}$ such that $T^{\prime}: F_{0}^{\prime} \rightarrow E^{\prime}$ is not absolutely summing. By [7, $\$ 4$, sec. 3] $T$ cannot be integral and so cannot be nuclear. If $J: F_{0} \rightarrow F$ is the inclusion map then $S=J T$ is not fully 
nuclear. Thus one of $E$ and $F$ must be finite dimensional. It is easy to see that a mapping to or from a finite-dimensional space is fully nuclear, completing the proof of the theorem.

REMARK II.4. If (C) is true as conjectured by Grothendieck, then if $\mathscr{L}(E, F)$ $=\mathrm{N}(E, F)$, it must be the case that $\mathscr{L}(E, F)=\mathrm{FN}(E, F)$. We will make further comments on this at the end of the chapter.

We now prove a result dual to Theorem II.3.

THEOREM II.5. Let E and $F$ be infinite-dimensional Banach spaces with $F$ isomorphic to a conjugate space. Then there is a quotient space $G$ of $F$ and a $T \in \mathscr{L}(E, G)$ such that $T$ is not nuclear.

Proof. By hypothesis, there is a Banach space $D$ such that $D^{\prime}$ is isomorphic to $F$. Let $D_{0}$ be a sufficiently Euclidean subspace of $D$. If $J: D_{0} \rightarrow D$ is the inclusion map then $J^{\prime}: D^{\prime} \rightarrow D_{0}^{\prime}$ is onto. Also we have an onto operator $S: F \rightarrow D_{0}^{\prime}$ (since $F$ is isomorphic to $D^{\prime}$ ). Since $D_{0}$, and hence $D_{0}^{\prime}$ is sufficiently Euclidean, as above, there is a nonnuclear operator from $E$ to $G=D_{0}^{\prime}$.

2. The linear and "ideal" structure of $\mathrm{FN}(E, F)$. It is well known [7], [13] that $\mathrm{N}(E, F)$ is a linear space and that if $S \in \mathscr{L}(E, F), T \in \mathrm{N}(F, G), R \in \mathscr{L}(G, H)$ then $T S \in \mathrm{N}(E, G)$ and $R T \in \mathrm{N}(F, H)$ for all Banach spaces $E, F, G$ and $H$.

Unfortunately $\mathrm{FN}(E, F)$ is not so well behaved.

Theorem II.6. Let $T \in \mathrm{N}(E, F)$ and suppose $\operatorname{Ker} T=T^{-1}(0)$ has infinite dimension. Then $T$ has a representation $T=T_{1}-T_{2}$ where $T_{1}, T_{2} \in \mathrm{FN}(E, F)$.

Proof. We may assume that $T$ is of the form $\sum_{i=1}^{\infty} f_{i} \otimes y_{i}$, where $\sum_{i=1}^{\infty}\left\|y_{i}\right\|<+\infty$ and $\left\|f_{i}\right\| \leqq C$. Choose $\left(x_{i}\right)$ in the kernel of $T$ and $\left(g_{i}\right)$ in $E^{\prime}$ such that $\left\|g_{i}\right\|=\left\|x_{i}\right\|=1$ and $g_{i}\left(x_{j}\right)=\delta_{i j}$. This is possible since $\operatorname{dim} \operatorname{Ker} T=+\infty$, by $(0.4)$. Let $T_{1}=\sum_{i=1}^{\infty}\left(f_{i}+g_{i}\right)$ $\otimes y_{i}$ and $T_{2}=\sum_{i=1}^{\infty} g_{i} \otimes y_{i}$. Since $x_{j} \in \operatorname{Ker} T$ for each $j$ and $g_{i}\left(x_{j}\right)=\delta_{i j}, T_{1}\left(x_{j}\right)$ $=\sum_{i=1}^{\infty}\left(f_{i}+g_{i}\right)\left(x_{j}\right) y_{i}=T x_{j}+y_{j}=y_{j} ;$ also, $T_{2}\left(x_{j}\right)=y_{j}$ and so $T_{1}, T_{2} \in \mathrm{FN}(E, F)$. Clearly $T=T_{1}-T_{2}$.

It follows from II.6 and the construction of Grothendieck (II.2(i)) that FN $(E, F)$ is not, in general, a linear space.

The ideal structure of $\mathrm{FN}(E, F)$ is also pathological. It is clear that $S T$ is fully nuclear whenever $T$ is fully nuclear. If $R \in \mathscr{L}(E, F)$ and the closure of $R(E)$ is $F$ then $T R$ is fully nculear whenever $T$ is. However, in general, $T R$ need not be fully nuclear whenever $T$ is. This fact is a consequence of the following: Every nuclear operator is the restriction of a fully nuclear operator. This result is proved in Chapter III.

3. A characterization of Hilbert spaces. Although $\mathrm{FN}(E, F)$ is not necessarily a linear space, there are very important cases where $\mathrm{FN}(E, F)$ is linear.

TheOREM II.7. Let $T: E \rightarrow F$ be nuclear and $\mathrm{cl}(T(E))$ complemented in $F$. Then $T$ is fully nuclear. 
Proof. Since $T$ is nuclear, there exists $\left\{x_{i}^{\prime}\right\} \subseteq E^{\prime},\left\{y_{i}\right\} \subseteq F, \sum_{i=1}^{\infty}\left\|x_{i}^{\prime}\right\| \cdot\left\|y_{i}\right\|<+\infty$ and $T x=\sum_{i=1}^{\infty}\left\langle x, x_{i}^{\prime}\right\rangle y_{i}$. If $P: E \rightarrow \operatorname{cl}(T(E))$ is a bounded projection, then $T x=P T x$ $=\sum_{i=1}^{\infty}\left\langle x, x_{i}^{\prime}\right\rangle P y_{i}$, and $\sum\left\|x_{i}^{\prime}\right\| \cdot\left\|P y_{i}\right\| \leqq\|P\| \sum_{i=1}^{\infty}\left\|x_{i}^{\prime}\right\| \cdot\left\|y_{i}\right\|<+\infty$ and it follows immediately that $T$ is fully nuclear.

Corollary II.8. If $F$ is isomorphic to a Hilbert space (in particular if $F$ is finite dimensional) then, for any Banach space $E, \mathrm{~N}(E, F)=\mathrm{FN}(E, F)$.

Proof. The proof is immediate from the theorem since every closed subspace of $F$ is complemented.

Since the mappings of finite rank are dense in $\mathrm{N}(E, F)$ with the nuclear norm [23], we see from II.8 that, for all Banach spaces $E$ and $F, \mathrm{FN}(E, F)$ is dense in $\mathrm{N}(E, F)$ (with the nuclear norm).

A partial converse to II.7 is also true.

THEOREM II.9. Let $F$ be a reflexive Banach space such that (i) each subspace of $F$ has the approximation property and (ii) if $T: E \rightarrow F$ is nuclear then $T$ is fully nuclear. Then every subspace of $F$ is complemented.

Proof. Let $F_{0}$ be a closed subspace of $F$ and $J$ the canonical map of $F_{0}^{\prime} \hat{\otimes} F_{0}$ into $F_{0}^{\prime} \hat{\otimes} F . J$ is one-to-one since $F_{0}$ has the approximation property [7, p. 165]. Moreover, $J$ is closed. If not, by the construction given in II.2(i), there would be a nuclear map $T: F_{0} \rightarrow F$ with $T\left(F_{0}\right) \subseteq F_{0}$ such that the astriction $T_{a}: F_{0} \rightarrow F_{0}$ is not nuclear, contradicting our hypothesis. Since $J$ is an isomorphism into, $J^{\prime}$ is onto. But $J^{\prime}: \mathscr{L}\left(F, F_{0}^{\prime \prime}\right) \rightarrow \mathscr{L}\left(F_{0}, F_{0}^{\prime \prime}\right)$ is restriction and since $F_{0}^{\prime \prime}=F_{0}$, the identity map, $I: F_{0} \rightarrow F_{0}$ has an extension $P: F \rightarrow F_{0}$ and $P^{2}=P$, thus $F_{0}$ is complemented.

REMARK II.10. It is clearly enough in II.9 to consider only reflexive Banach spaces $E$. This observation will be useful later.

If $H$ is a Hilbert space, and $F$ is a closed subspace of $H$, and $E$ is any Banach space then the canonical map $J: E^{\prime} \hat{\otimes} F \rightarrow E^{\prime} \hat{\otimes} H$ is always an isometry.

The converse is also true.

THEOREM II.11. Suppose $F$ is a Banach space satisfying the conditions of II. 6 and with the following property: the nuclear norm in $E^{\prime} \hat{\otimes} \mathrm{cl}(T(E))$ is the same as in $E^{\prime} \hat{\otimes} F$. Then $F$ is isometric to a Hilbert space.

Proof. As above, the canonical map $J: F_{0}^{\prime} \hat{\otimes} F_{0} \rightarrow F_{0}^{\prime} \hat{\otimes} F$ is an isometry whenever $F_{0}$ is a closed subspace of $F$. Thus the adjoint map $J^{\prime}: \mathscr{L}\left(F, F_{0}\right) \rightarrow \mathscr{L}\left(F_{0}, F_{0}\right)$ is onto, and by the Hahn-Banach theorem, since $J$ is an isometry, there is an operator $P: F \rightarrow F_{0}$ such that $P$ restricted to $F_{0}$ is the identity and $\|P\|=1$. Thus by Kakutani's theorem [14], $F$ is isometric to a Hilbert space.

The following remarks pertain to II.9 and II.11.

REMARK II.12. (i) Grothendieck [7, §5, sec. 2] has conjectured that all reflexive Banach spaces have the approximation property, so part (i) of the hypothesis of Theorem II.9 is probably superfluous. 
(ii) We have not used the full strength of hypothesis (ii) in II.9 in proving II.9 or II.11 since we used the properties only for subspaces of $F$. This does, however, give an easy, although apparently new, characterization of Hilbert space.

(iii) Using the construction of II.2(i) and a recent result of Rosenthal we show how exacting II.9 and II.11 actually are. By Rosenthal [26, p. 52] there is an uncomplemented subspace of $L_{4 / 3}$ which is isomorphic to $l_{2}$. Let $S: l_{2} \rightarrow L_{4 / 3}$ be such an into isomorphism. It follows as in II.2(i) that $I \otimes S: l_{2} \hat{\otimes} l_{2} \rightarrow l_{2} \hat{\otimes} L_{4 / 3}$ is not closed, that is, there is an operator $T: l_{2} \rightarrow l_{2}$ that is not nuclear, but $S T: l_{2} \rightarrow L_{4 / 3}$ is nuclear. This shows how important (ii) in the hypothesis of II.9 is.

(iv) If $S$ and $T$ have the meaning as in II.7(iii) then as just observed, $S T$ is nuclear, that is, we may compose a nonnuclear operator $T$ with an into isomorphism $S$ to obtain a nuclear operator. In [23, p. 49], [25] Pietsch introduced the idea of a quasi-nuclear operator and proved that if $T: E \rightarrow F$ is quasi-nuclear, then if $J: F \rightarrow l_{\infty}(S)$ is the canonical operator where $S$ is the unit ball of $F^{\prime}$, then $J T$ is nuclear [23, p. 52] (cf. 0.16). Thus the situation in (iii) is actually quite common. In fact, it is interesting to note that the spaces in (iii) are reflexive. A large class of examples is given in the next chapter.

(v) It should be mentioned that II.9 may actually characterize Hilbert space. It is still an open question as to whether the conclusion of II.9 characterizes Hilbert $\operatorname{space}\left({ }^{4}\right)$.

4. An analog of a problem of Grothendieck. It is easily seen that if $T: E \rightarrow F$ is nuclear, then $T^{\prime}: F^{\prime} \rightarrow E^{\prime}$ is nuclear [23, p. 48]. The converse question is still open. ([23]; some partial results are given in [7].) The analogous problem for fully nuclear operators is solved in the following theorem.

THEOREM II.13. There is a nonfully nuclear operator $T$ whose adjoint $T^{\prime}$ is fully nuclear.

Proof. Let $E$ be a sufficiently Euclidean subspace of $l_{1}$, with a basis, which exists by Theorem I.15. Again we use the technique of II.2(i). The canonical map $J: E \hat{\otimes} l_{2} \rightarrow l_{1} \otimes l_{2}$ is one-to-one, since $l_{2}$ has the approximation property $[7, \S 5$, sec. 3]. However, $J$ is not closed because if it were, the adjoint $J^{\prime}: \mathscr{L}\left(l_{1}, l_{2}\right) \rightarrow$ $\mathscr{L}\left(E, l_{2}\right)$ which is restriction would be onto. Every operator from $l_{1}$ to $l_{2}$ is absolutely summing [8], [18] and so the restriction of such an operator is absolutely summing. By Theorem I.10 there exists a nonabsolutely summing operator from $E$ to $l_{2}$. Thus $E \hat{\otimes} l_{2}$ is not closed in $l_{1} \hat{\otimes} l_{2}$. Choose $T$ in the closure of $E \hat{\otimes} l_{2}$ in $l_{1} \hat{\otimes} l_{2}$ but not in $E \hat{\otimes} l_{2}$. Regarding $T$ as an operator from $l_{2}$ to $l_{1}$ with $T\left(l_{2}\right) \subseteq E, T$ is nuclear but not fully nuclear. Anticipating a result in Chapter III we claim that $T^{\prime}$ is fully nuclear.

$\left.{ }^{4}\right)$ This long outstanding problem has been solved affirmatively by J. Lindenstrauss and L. Tzafriri, On the complemented subspace problem, Israel J. Math. 9 (1971), 263-269. Thus our II. 9 characterizes the isomorphs of Hilbert space. 
5. Questions. In Chapter II we have given criteria for full nuclearity only in terms of the range. In Chapter III we consider criteria in terms of the domain and obtain a rather surprising theorem.

The unsolved problems concerning Chapter II appear to be extremely difficult. The most obvious question is the following:

(Q5) If there is a nonfully nuclear operator $T$ from $E$ to $F$, is there a nonnuclear operator from $E$ to $F$ ?

As mentioned earlier in connection with II.9, the problems concerning II.9 and II.11 are classical and we will not attempt to discuss the difficulties $\left({ }^{4}\right)$.

In reference to (Q5) we do feel that the material developed in Chapter I can eventually be expanded to prove (C) exactly as asserted by Grothendieck.

\section{Characterizations of $\mathscr{L}_{\infty}$-Spaces}

1. $\mathscr{L}_{\infty}$-spaces. To motivate this chapter we first make an easy, though interesting, observation which seems to have received little attention.

THEOREM III.1. Let $F$ be any Banach space, then any absolutely summing map $T: c_{0} \rightarrow F$ is nuclear. In particular, any nuclear map from $c_{0}$ to $F$ is fully nuclear $\left(^{5}\right)$.

Proof. Let $\left(e_{i}\right)$ denote the usual unit vector basis of $c_{0}$. The formal series $\sum_{i=1}^{\infty} e_{i}$ is weakly unconditionally Cauchy, and thus $\sum_{i=1}^{\infty}\left\|T e_{i}\right\|<+\infty$ [23, p. 36]. If $x=\sum_{i=1}^{\infty} f_{i}(x) e_{i}$ in $c_{0}$, then $T x=\sum_{i=1}^{\infty} f_{i}(x) T e_{i}$, where $\left(f_{i}\right)$ is the unit vector basis of $l_{1}$. Also, $\sum_{i=1}^{\infty}\left\|f_{i}\right\| \cdot\left\|T e_{i}\right\|=\sum_{i=1}^{\infty}\left\|T e_{i}\right\|<+\infty$, which implies $T$ is nuclear. Also, if $T: c_{0} \rightarrow F$ is nuclear, then it is absolutely summing, so the astriction $T_{a}: c_{0} \rightarrow$ $\mathrm{cl}\left(T\left(c_{0}\right)\right)$ is certainly absolutely summing, and by the above, nuclear. Thus $T$ is fully nuclear.

REMARK III.2. (i) It is easily observed that the above theorem generalizes to $c_{0}(S)$ spaces, $S$ any set.

(ii) Grothendieck [8] has observed that the canonical operator from $C[0,1]$ to $L_{1}[0,1]$ is integral (hence absolutely summing) but not nuclear. By the profound result of Miljutin [20] it follows that if $K$ is compact metric and uncountable then there is a nonnuclear, integral operator $T: C(K) \rightarrow L_{1}[0,1]$.

(iii) More generally, the canonical operator $L_{\infty}(S, \mu) \rightarrow L_{1}(S, \mu), S$ a locally compact Hausdorff space, $\mu$ a measure on $S$ [30, Exposés 3, 4, 16] is integral, but not, in general, nuclear (not in general, even compact) [30, Exposé 16, p. 5]. In particular, the operator $L_{\infty}[0,1] \rightarrow L_{1}[0,1]$ is integral but not nuclear. Moreover, $l_{\infty}$ is isomorphic to $L_{\infty}[0,1]$ [18]. Since $l_{\infty}$ is isometrically, isomorphic to $C(\beta N)$, the Stone-Čech compactification of the positive integers $N$, there is an integral, nonnuclear operator from $C(\beta N)$ to $L_{1}[0,1]$. It follows from a recent result of Rosenthal [27] that if $K$ is any Stonian space (i.e., compact, Hausdorff and the

$\left({ }^{5}\right)$ The following generalizations of III.1 and III.7 have been proved by D. R. Lewis and C. Stegall: If $E$ is a Banach space then $E^{\prime}$ is isomorphic to $l_{1}(\Gamma)$ if and only if $\operatorname{AS}(E, F)$ $=\mathrm{N}(E, F)$ for all Banach spaces $F$ (paper submitted to J. Functional Analysis). 
closure of every open set is again open) then there is a nonnuclear, integral operator from $C(K)$ to $L_{1}[0,1]$.

The second part of Theorem III.1 is true for any $\mathscr{L}_{\infty}$-space. We were led to this result by the fact that the technique of II.2(i) fails because the dual of an $\mathscr{L}_{\infty}$-space is an $\mathscr{L}_{1}$-space, and the dual of an $\mathscr{L}_{1}$-space is an injective space [15], [18], [19].

THEOREM III.3. The following assertions are equivalent:

(i) $\mathrm{I}(E, F)=\mathrm{AS}(E, F)$ for all Banach spaces $F$;

(ii) $\mathrm{N}(E, F)=\mathrm{QN}(E, F)$ for all Banach spaces $F$;

(iii) $\mathrm{N}(E, F)=\mathrm{FN}(E, F)$ for all Banach spaces $F$; and

(iv) $E$ is an $\mathscr{L}_{\infty}$-space.

Proof. (i) $\rightarrow$ (ii). Let $T \in \mathrm{QN}(E, F)$. By 0.14 and 0.17 we have the following diagram:

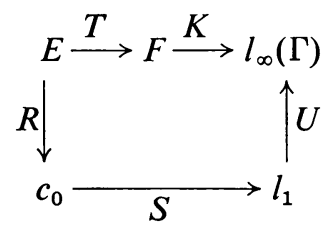

Now $S R$ is nuclear and so the astriction $(S R)_{a}$ is absolutely summing. By (i), $(S R)_{a}$ is integral. Let $U_{0}$ denote the restriction $U$ to $\mathrm{cl}(S R(E))$. Since $U$ and hence $U_{0}$ is compact

$$
U_{0}(S R)_{a}: E \rightarrow \operatorname{cl}(U(S R(E)))=\operatorname{cl}(K T(E))=\operatorname{cl}(K(T(E)))
$$

is nuclear and so $K^{-1} U_{0}(S R)_{a}: E \rightarrow \operatorname{cl}(T(E))$ is nuclear, i.e. $T$ is nuclear.

(ii) $\rightarrow$ (iii). Since the astriction of a nuclear operator is clearly quasi-nuclear, this implication is trivial.

(iii) $\rightarrow$ (iv). Suppose $F$ is a Banach space with the approximation property and $F_{0}$ is a closed subspace of $F$. Consider the diagram

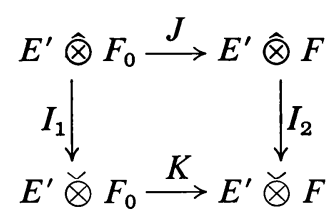

Since $F$ has the approximation property $I_{2}$ is one-to-one; $K$ is always one-to-one hence $\operatorname{Ker} J=\operatorname{Ker} I_{1}$. Now, Ker $I_{1}=\left\{u:\left\langle u x, y^{\prime}\right\rangle=0, x \in E, y^{\prime} \in F_{0}^{\prime}\right\}, u=\sum_{i=1}^{\infty} \lambda_{i} x_{i}^{\prime}$ $\otimes y_{i}$. Regarding $u$ as an operator, $u(x)=0$ for all $x$ then $u^{\prime \prime}\left(x^{\prime \prime}\right)=0$ for all $x^{\prime \prime} \in E^{\prime \prime}$ since $u$ (and thus $u^{\prime \prime}$ ) as an operator is 0 . 
From this observation and the fact that $F$ has the approximation property (and by (iii)) $E^{\prime} \hat{\otimes} F_{0} \stackrel{J}{\rightarrow} E^{\prime} \hat{\otimes} F$ is closed. Moreover, we have the diagrams:

$$
\begin{gathered}
E^{\prime} \hat{\otimes} F_{0} \longrightarrow E^{\prime} \hat{\otimes} F \\
E^{\prime} \hat{\otimes} F_{0} / \operatorname{Ker} J
\end{gathered}
$$

and

$(++)$

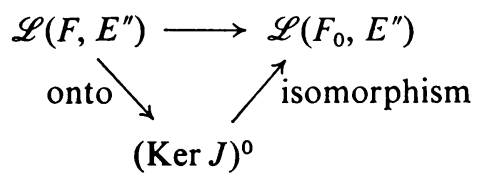

It is easy to see that $(\operatorname{Ker} J)^{0}$ contains the operators of finite rank from $F_{0}$ to $E^{\prime \prime}$.

Let $\left\{F_{\alpha}\right\}$ be the collection of all nontrivial finite-dimensional subspaces of $l_{\infty}$ and let $F_{0}=\left(\sum_{\alpha \in \Gamma} \oplus F_{\alpha}\right)_{l_{\infty}}$ and $F=\left(\sum_{\alpha \in \Gamma} \oplus l_{\infty}\right)_{l_{\infty}}$ for a suitable index set $\Gamma$. Since $F$ is isometrically isomorphic to $l_{\infty}(\Gamma \times N), N$ the positive integers, $F$ has the approximation property. Now by (iii), $E^{\prime} \hat{\otimes} F_{0} \rightarrow E^{\prime} \hat{\otimes} F$ is closed. Let $G_{1} \subseteq G_{2}, \operatorname{dim} G_{2}<+\infty$. Thus there exists $G \subset l_{\infty}$ with $G$ isometrically isomorphic to $G_{2}$ and an $\alpha$ such that $F_{\alpha} \subseteq G \subseteq l_{\infty}$ and $F_{\alpha}$ isometrically isomorphic to $G_{1}$. Let $S: F_{\alpha} \rightarrow E^{\prime \prime}$ and $P_{\alpha}: F_{0} \rightarrow F_{\alpha}$, $P_{\alpha}$ a projection of norm 1 . Then $S P_{\alpha}: F_{0} \rightarrow E^{\prime \prime}$ is of finite rank. By the diagram $(++)$ the restriction operator $\mathscr{L}\left(F, E^{\prime \prime}\right) \rightarrow(\operatorname{Ker} J)^{0}$ is onto and so by the open mapping theorem there is a $C>0$ such that for $S \in(\operatorname{Ker} J)^{0}$ there is an extension $\tilde{S} \in \mathscr{L}\left(F, E^{\prime \prime}\right)$ such that $\|\tilde{S}\| \leqq C\|S\|$. Thus there is an operator $\tilde{S}: F \rightarrow E^{\prime \prime}$ with $\tilde{S} \mid F_{0}=S P_{\alpha},\|\tilde{S}\| \leqq C\left\|S P_{\alpha}\right\| \leqq C\|S\|$. Now restrict $\tilde{S}$ to the $\alpha$ th factor of $\left(\sum_{\beta \in \Gamma} \oplus l_{\infty}\right)_{l_{\infty}}$ and restrict again to $G$ to obtain

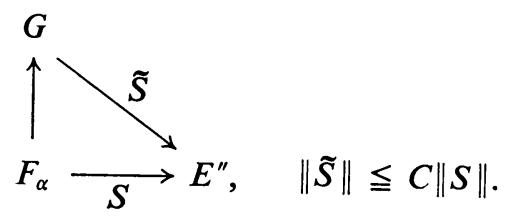

By a remark of Lindenstrauss and Rosenthal [19], $E^{\prime \prime}$ is injective. Hence by [19], $E$ is an $\mathscr{L}_{\infty}$-space.

(iv) $\rightarrow$ (i). Suppose $E$ is an $\mathscr{L}_{\infty, \lambda}$-space and $T \in \operatorname{AS}(E, F)$. Let $T_{B}: E \times F^{\prime} \rightarrow C$ be the induced bilinear form, i.e. $T_{B}\left(x, y^{\prime}\right)=\left\langle T x, y^{\prime}\right\rangle$. The idea of the proof is to show that $T_{B}: E \check{\otimes} F^{\prime} \rightarrow C$ is continuous. To this end let $\sum_{i=1}^{n} x_{i} \otimes y_{i}^{\prime} \in E \otimes F^{\prime}$ with $\left\|\sum_{i=1}^{n} x_{i} \otimes y_{i}^{\prime}\right\|_{\varepsilon} \leqq 1$. Since $E$ is an $\mathscr{L}_{\infty, \lambda}$-space there is a finite-dimensional space $X$ with $\left[x_{i}: i \leqq n\right] \subseteq X \subset E$ with $d\left(X, l_{\infty}^{m}\right)<\lambda+1$ where $m=\operatorname{dim} X$. Suppose 
$S: l_{\infty}^{m} \rightarrow X$ is such that $\|S\|=1,\left\|S^{-1}\right\| \leqq \lambda+1$, and $z_{j}=S\left(e_{j}\right)$ where $\left(e_{j}\right)$ is the unit vector basis of $l_{\infty}^{m}$. Now $x_{i}=\sum_{j=1}^{m} t_{i j} z_{j}, 1 \leqq i \leqq n$, and so

i.e.

$$
\begin{aligned}
1 & \geqq \operatorname{Sup}\left\{\left|\sum_{i=1}^{n} \sum_{j=1}^{m} t_{i j}\left\langle z_{j}, x^{\prime}\right\rangle\left\langle y, y_{i}^{\prime}\right\rangle\right|:\left\|x^{\prime}\right\|=\|y\|=1\right\} \\
& =\operatorname{Sup}\left\{\left\|\sum_{j=1}^{m}\left(\sum_{i=1}^{n} t_{i j}\left\langle y, y_{i}^{\prime}\right\rangle\right) z_{j}\right\|:\|y\|=1\right\} \\
& \geqq(\lambda+1)^{-1} \operatorname{Sup}\left\{\max _{1 \leqq j \leqq m}\left|\sum_{i=1}^{n} t_{i j}\left\langle y, y_{i}^{\prime}\right\rangle\right|:\|y\|=1\right\} \\
& =(\lambda+1)^{-1} \max _{1 \leqq j \leqq m}\left\|\sum_{i=1}^{n} t_{i j} y_{i}^{\prime}\right\|
\end{aligned}
$$

$$
\max _{1 \leqq j \leqq m}\left\|\sum_{i=1}^{n} t_{i j} y_{i}^{\prime}\right\| \leqq \lambda+1 \text {. }
$$

On the other hand since $T$ is absolutely summing we have

$$
\begin{aligned}
\sum_{j=1}^{m}\left\|T z_{i}\right\| & \leqq\|T\|_{\text {as }} \operatorname{Sup}\left\{\sum_{j=1}^{m}\left|\left\langle z_{j}, x^{\prime}\right\rangle\right|:\left\|x^{\prime}\right\|=1\right\} \\
& =\|T\|_{\text {as }} \operatorname{Sup}\left\{\sum_{j=1}^{m}\left|\left\langle S e_{j}, x^{\prime}\right\rangle\right|:\left\|x^{\prime}\right\|=1\right\} \\
& =\|T\|_{\text {as }} \operatorname{Sup}\left\{\sum_{j=1}^{m}\left|\left\langle e_{j}, S^{\prime} x^{\prime}\right\rangle\right|:\left\|x^{\prime}\right\|=1\right\} \\
& \leqq\|T\|_{\text {as }} \operatorname{Sup}\left\{\sum_{j=1}^{m}\left|\left\langle e_{j}, l\right\rangle\right|:\|l\| \leqq 1, l \in l_{1}^{m}\right\}=\|T\|_{\text {as }}
\end{aligned}
$$

i.e.

(2)

$$
\sum_{j=1}^{m}\left\|T z_{j}\right\| \leqq\|T\|_{\text {as }}
$$

Combining (1) and (2) we have

$$
\begin{aligned}
\left|\left\langle\sum_{i=1}^{n} x_{i} \otimes y_{i}^{\prime}, T_{B}\right\rangle\right| & =\left|\sum_{i=1}^{n}\left\langle T x_{i}, y_{i}^{\prime}\right\rangle\right|=\left|\sum_{i=1}^{n} \sum_{j=1}^{m} t_{i j}\left\langle T z_{i}, y_{i}^{\prime}\right\rangle\right| \\
& =\left|\sum_{j=1}^{m}\left\langle T z_{j}, \sum_{i=1}^{n} t_{i j} y_{i}^{\prime}\right\rangle\right| \\
& \leqq \sum_{j=1}^{m}\left\|T z_{j}\right\|\left\|\sum_{i=1}^{n} t_{i j} y_{i}^{\prime}\right\| \leqq(\lambda+1)\|T\|_{\text {as. }}
\end{aligned}
$$

Thus $T_{B}$ is continuous and so $T \in J\left(E, F^{\prime}\right)=\mathrm{I}\left(E, F^{\prime \prime}\right)$. It follows that $T$ is integral.

It is immediate from III.2(i) and 0.15 , that an absolutely summing operator with $\mathscr{L}_{\infty}$-domain and reflexive range is nuclear.

ReMARK III.4. (i) Theorem III.3 was originally proved with the additional hypothesis that $E^{\prime}$ have the approximation property in (i), (ii) and (iii). This hypothesis was used in (iii) $\rightarrow$ (iv) to assert that $J: E^{\prime} \hat{\otimes} F_{0} \rightarrow E^{\prime} \hat{\otimes} F$ was one-toone. It is interesting that (iv) implies that $E^{\prime}$ has the approximation property. 
That $E^{\prime}$ has the approximation property follows from these observations: Since $E$ is an $\mathscr{L}_{\infty}$-space $E^{\prime \prime}$ is injective, hence complemented in $l_{\infty}(\Gamma)$ for some $\Gamma$. But $l_{\infty}(\Gamma)$ has the metric approximation property and so to show that $E^{\prime}$ has the approximation property it suffices [30] to show that $E^{\prime \prime}$ has the approximation property; this fact is well known. It has been pointed out to us by Professor A. Lazar that the approximation property hypothesis can also be deleted by making the following observation: Suppose a Banach space $E$ has the following property: For all Banach spaces $Y, Z$ with $Y \subset Z$, the restriction map $\gamma: \mathscr{L}(Z, E) \rightarrow \mathscr{L}(Y, E)$ has closed range. Then $E$ is an $\mathscr{L}_{\infty}$-space.

Indeed, if the property holds there is a $\lambda$ such that for every $T \in \gamma(\mathscr{L}(Z, E))$ there is a $\tilde{T} \in \mathscr{L}(Z, E)$ with $\tilde{T} \mid Y=T$ and $\|\tilde{T}\| \leqq \lambda\|T\|$. For if not, there are Banach spaces $Y_{n} \subset Z_{n}$, operators $T_{n} \in \gamma\left(\mathscr{L}\left(Z_{n}, E\right)\right),\left\|T_{n}\right\|=1$, such that for any extension $\tilde{T}_{n} \in \mathscr{L}\left(Z_{n}, E\right),\left\|\tilde{T}_{n}\right\| \geqq n^{3}$. Let $Y=\left(Y_{1} \oplus Y_{2} \oplus \cdots\right)_{c_{0}}$ and $Z=\left(Z_{1} \oplus Z_{2} \oplus \cdots\right)_{c_{0}}$.

Define $T: Y \rightarrow E$ by $T y=\sum_{n=1}^{\infty}\left(1 / n^{2}\right) T_{n}\left(y_{n}\right)$ where $y=\left(y_{n}\right)$. Since $T$ is the limit of extendable operators and $\gamma$ has a closed range, there is an extension $\tilde{T}: Z \rightarrow E$, of $T$. Choose $n>\|\tilde{T}\|$. Now $n^{2} \widetilde{T} \mid Z_{n}$ is an extension of $T_{n}$ and $\left\|n^{2} \tilde{T} \mid Z_{n}\right\| \leqq n^{2}\|\tilde{T}\|<n^{3}$, a contradiction. Thus, such a $\lambda$ exists (this argument is essentially due to Goodner [36]).

This fact applied to finite-dimensional $Y, Z$ together with the remark of Lindenstrauss and Rosenthal [19] used earlier imply that $E^{\prime \prime}$, hence $E$, is an $\mathscr{L}_{\infty}$-space.

To see how this applies to (iii) $\rightarrow$ (iv) observe that for finite-dimensional $F$, $I_{1}$ is closed, hence $\gamma=I_{1}^{\prime}$ is closed, yielding the desired result.

(ii) Let us mention that using the above one can add the following statements to the list of equivalences in III.2 (a fully integral map, denoted $T \in \mathrm{FI}(E, F)$, is defined analogously to that of a fully nuclear map):

(a) $\mathrm{I}(E, F)=\mathrm{FI}(E, F)$ for all Banach spaces $F$;

(b) $\mathrm{N}(E, F)=\mathrm{FN}(E, F)$ for all conjugate Banach spaces $F$;

(c) $\mathrm{N}(E, F)=\mathrm{FN}(E, F)$ for all reflexive Banach spaces $F$;

(d) and (e) same as (b) and (c) replacing "nuclear" by "integral".

Indeed the nontrivial implication can be deduced from the proof of III.3.

(iii) It is curious, and somewhat surprising, that when an $\mathscr{L}_{\infty}$-space is a range space that the same phenomenon of III.2 occurs. More precisely, we have the following theorem:

The following are equivalent:

(a) $E$ is an $\mathscr{L}_{\infty}$-space;

(b) $\mathrm{AS}(F, E)=\mathrm{I}(F, E)$ for all Banach spaces $F$;

(c) $\mathrm{QN}(F, E)=\mathrm{N}(F, E)$ for all Banach spaces $F$;

(d) $\mathrm{QN}(F, E)=\mathrm{N}(F, E)$ for all Banach spaces $F$ such that $F^{\prime \prime}$ has the approximation property.

Proof. (a) $\rightarrow$ (b). If $E$ is an $\mathscr{L}_{\infty}$-space then $E^{\prime \prime}$ is injective [19]. By 0.12 , if $T \in \operatorname{AS}(F, E)$ then $T^{\prime \prime} \in \operatorname{AS}\left(F^{\prime \prime}, E^{\prime \prime}\right)$. We claim that $T^{\prime \prime}$ is integral. To see this 
observe that, by $0.12, T$ factors through a subspace of an $L_{1}(\mu)$-space for some positive measure $\mu$. Since $E^{\prime \prime}$ is injective it follows that $T^{\prime \prime}$ factors through the canonical embedding of $C(K) \rightarrow L_{1}(\mu)$ which is integral [7], [8]. Thus $T^{\prime \prime}$, hence $T$, is integral [7].

(b) $\rightarrow$ (d). Let $F$ be a Banach space such that $F^{\prime \prime}$ has the approximation property and let $T: F \rightarrow E$ be quasi-nuclear and let $I: E \rightarrow l_{\infty}(\Gamma)$ be the canonical injection. Then $I T$ is nuclear [25] and we have the factorization of Theorem 0.14:

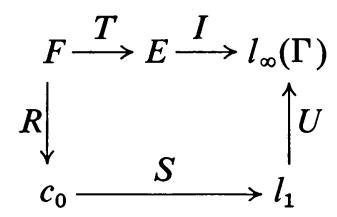

From this we obtain the following factorization:

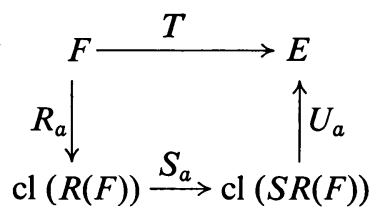

Since $S_{a}$ is absolutely summing, $U_{a} S_{a}$ is absolutely summing, and by (b) is integral. Since $R_{a}$ is compact and $F^{\prime \prime}$ has the approximation property $U_{a} S_{a} R_{a}=T$ is nuclear [7, Theorem 10, Proposition 15].

(d) $\rightarrow$ (a). The proof is similar to that of (iii) $\rightarrow$ (iv) of III.3. Let $F=\left(\sum \oplus E_{\alpha}\right)_{l_{2}}$ where $E_{\alpha} \subseteq l_{\infty}$ and $\operatorname{dim} E_{\alpha}<+\infty$. It is obvious that $F$ is reflexive and has the approximation property. By (d) it follows that $J: F^{\prime} \hat{\otimes} E \rightarrow F^{\prime} \hat{\otimes} G$ is an isomorphism for any space $G$ containing $E$. Thus $J^{\prime}: \mathscr{L}(G, F) \rightarrow \mathscr{L}(E, F)$ is onto and by the open mapping theorem there is a $C>0$ such that if $T \in \mathscr{L}(E, F)$ then there exists a $\tilde{T}$ in $\mathscr{L}(G, F),\|\tilde{T}\| \leqq C\|T\|$, such that $J^{\prime}(\tilde{T})=T$, that is, $T$ has an extension $\tilde{T}: G \rightarrow F$. If $T(E) \subseteq E_{\alpha}$ for some $\alpha$ then we may compose $T$ with the canonical projection $P_{\alpha}: F \rightarrow E_{\alpha},\left\|P_{\alpha}\right\|=1$, and we have an extension of $T, P_{\alpha} \tilde{T}: G \rightarrow E_{\alpha}$. By repeating the argument above we see that the constant $C$ is independent of $G$. Thus by [19, Remark 1 after Theorem 4.1] $E$ is an $\mathscr{L}_{\infty}$-space.

Since (c) $\rightarrow$ (d) is obvious we need only prove (a) $\rightarrow$ (c). Suppose $T: F \rightarrow E$ is quasi-nuclear and $E$ is an $\mathscr{L}_{\infty}$-space. Suppose $I: E \rightarrow l_{\infty}(\Gamma)$ is the canonical injection. Then the composition $I T$ is nuclear and we have the factorization of Theorem 0.14

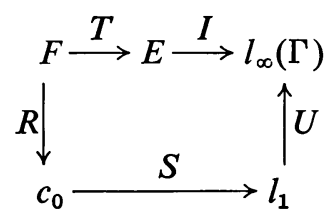


from which we obtain the following factorization:

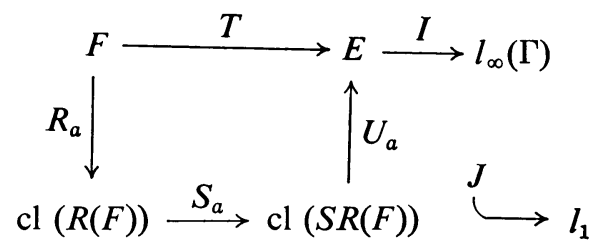

Since $U_{a}$ is compact and $E$ is an $\mathscr{L}_{\infty}$-space there exists an extension $\widetilde{U}_{a}: l_{1} \rightarrow E$ [19]. Since $J S_{a}$ is nuclear and $T=\widetilde{U}_{a} J S_{a} R_{a}$ we have that $T$ is nuclear.

There are numerous corollaries to III.3. Perhaps the most important is the following result of [19].

COROLlaRY III.5. A complemented subspace of an $\mathscr{L}_{\infty}$-space is an $\mathscr{L}_{\infty}$-space.

Proof. Let $X$ be a complemented subspace of an $\mathscr{L}_{\infty}$-space $E$ and $F$ an arbitrary Banach space. Let $T \in N(X, F)$ and $P: E \rightarrow X$ a projection. By III.3, TP is fully nuclear and, since $P$ is onto, $T$ is fully nuclear and so, by III.3, $X$ is an $\mathscr{L}_{\infty}$-space.

The main examples of quasi-nuclear operators which are not nuclear are the nonnuclear Hilbert-Schmidt operators between Hilbert spaces [24]. The existence of such operators for general Banach spaces appears not to have been studied. Thus the following corollary is interesting.

Corollary III.6. If $E$ is not an $\mathscr{L}_{\infty}$-space, then there exists a Banach space $F$, and a quasi-nuclear operator $T: E \rightarrow F$ that is not nuclear.

COROllary III.7. Suppose $E$ has an unconditional basis. Then $E$ is isomorphic to $c_{0}$ if and only if, for each Banach space $F, \mathrm{AS}(E, F)=\mathrm{N}(E, F)$.

Proof. If $\mathrm{AS}(E, F)=\mathrm{N}(E, F)$ then certainly $\mathrm{QN}(E, F)=\mathrm{N}(E, F)$, and by Corollary III.6, $E$ is isomorphic to an $\mathscr{L}_{\infty}$-space and from [18, Corollary 5, p. 297] it follows that $E$ must be isomorphic to $c_{0}$. The converse is just III.1.

COROllary III.8. If $E$ is isomorphic to a quotient space of a $\mathscr{L}_{\infty}$-space $G$, and $F$ is any Banach space, then there is an operator $T: E \rightarrow F$ that is not nuclear.

(We remark that III.8 is valid for $E$ a quotient of an $\mathscr{L}_{\infty}$-space or a subspace of an $\mathscr{L}_{1}$-space or $F$ a quotient of an $\mathscr{L}_{\infty}$-space or a subspace of an $\mathscr{L}_{1}$-space.)

Proof. Construct $T: E \rightarrow F$ as in Theorem I.22. Let $S: G \rightarrow E$ be onto. If $T$ is nuclear, then $T S: G \rightarrow F$ is nuclear, hence it is fully nuclear. It follows that the dual $(T S)_{a}^{\prime}: F_{0}^{\prime} \rightarrow G^{\prime}$ of the astriction $(T S)_{a}: G \rightarrow F_{0}$ is nuclear. But $\mathrm{cl}(T(E))=F_{0}$ is sufficiently Euclidean and it follows by the construction of I.20 that $(T S)_{a}^{\prime}$ is not even absolutely summing.

COROLLARY III.9. Every nuclear operator is the restriction of a fully nuclear operator. 
Proof. Let $T: E \rightarrow F$ be nuclear. Let $S$ be the unit ball of $E^{\prime}$, and consider the canonical injection $J: E \rightarrow l_{\infty}(S)$. Then there exists a nuclear operator $\tilde{T}: l_{\infty}(S) \rightarrow F$ such that $T=\tilde{T} J . \tilde{T}$ is fully nuclear, and $T$ is the restriction of $\tilde{T}$.

This is the result promised in II.13.

Corollary III.10. Let $T: E \rightarrow F$ be nuclear such that $\operatorname{dim} T(E)$ is infinite. Then there exists an infinite-dimensional subspace $E_{0}$ of $E$ such that the restriction of $T$, $T_{0}: E_{0} \rightarrow F$, is fully nuclear.

Proof. Suppose we represent $T$ as $\sum_{i=1}^{\infty} \lambda_{i} f_{i} \otimes y_{i},\left\|f_{i}\right\| \rightarrow 0,\left\|y_{i}\right\| \rightarrow 0, \sum_{i=1}^{\infty}\left|\lambda_{i}\right|$ $<+\infty$. Then consider the factorization of Theorem II.11:

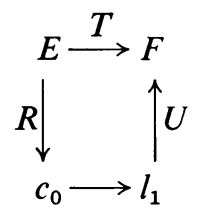

Thus, $R(E)$ is a separable infinite-dimensional subspace of $c_{0}$, so $\mathrm{cl}(R(E))$ contains a complemented subspace $G$ isomorphic to $c_{0}$ [21], [31]. Let $P: \mathrm{cl}(R(E)) \rightarrow G$ be a projection. Let $E_{0}=R^{-1}(G), E_{0}$ is closed since $G$ is closed, and $E_{0}$ is infinite dimensional since $R\left(E_{0}\right)$ is dense in $G$. Let $T_{0}: E_{0} \rightarrow F, R_{0}: E_{0} \rightarrow G,(U S)_{0}: G \rightarrow F$ be the appropriate restrictions and astrictions. $U S$ is nuclear, so $(U S)_{0}$ is nuclear, and $G$ is isomorphic to $c_{0}$, so $(U S)_{0}: G \rightarrow F$ is fully nuclear. But $R_{0}\left(E_{0}\right)$ is dense in $G$ so, $(U S)_{0} R_{0}: E_{0} \rightarrow F$ is fully nuclear but $\operatorname{cl}\left(T\left(E_{0}\right)\right)=\operatorname{cl}\left((U S)_{0} R_{0}\left(E_{0}\right) T_{0}\left(E_{0}\right)\right)$. Thus $T_{0}$ is fully nuclear.

2. Questions. We have made most of the comments concerning $\mathscr{L}_{\infty}$-spaces and their operator characterization in the preceding pages. The following question would advance the theory put forth in Chapter III:

(Q6) Suppose $E$ is an $\mathscr{L}_{\infty}$-space. Does the restriction map $\gamma: \mathscr{L}(Z, E) \rightarrow \mathscr{L}(Y, E)$ have a closed range for all pairs $\langle Y, Z\rangle, Z \supset Y$ ? (Converse of III.4(i).)

As mentioned earlier, (Q6) was posed by A. Lazar.

\section{COMPletely Nuclear Operators}

1. Introduction. In this chapter we consider the notion dual to that of a fully nuclear operator. As to be expected, our results parallel those of Chapter II.

Definition IV.1. Let $T \in \mathscr{L}(E, F)$. We say that $T$ is completely nuclear and write $T \in \mathrm{CN}(E, F)$ if $T_{K}$, defined by

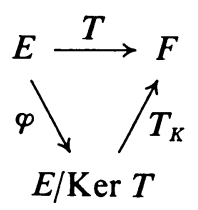

is nuclear, where $\varphi$ denotes the canonical mapping: $E \rightarrow E / \operatorname{Ker} T$. It will follow 
from the elementary fact below that, in general, nuclear operators are not completely nuclear.

2. Duality with fully nuclear operators. Let us recall that for $T \in \mathscr{L}(E, F), T_{a}$ denotes the astriction operator $T_{a}: E \rightarrow \mathrm{cl}(T(E))$.

REMARK IV.2. We have the following duality (for arbitrary operators and their adjoints):

(a) $\left[T_{a}\right]^{\prime}=\left[T^{\prime}\right]_{K}$;

(b) $\left[T_{K}\right]^{\prime}=\left[T_{a}\right]^{\prime} \psi$ where $\psi$ is canonical: $F^{\prime} \rightarrow F^{\prime} / \mathrm{cl}\left(T(E)^{0}\right)$;

(c) if $E$ is reflexive, $\left[T^{\prime}\right]_{a}=\left[T_{K}\right]^{\prime}$.

These facts are immediate from the relationships between images and annihilators of operators and their adjoints; see e.g. [43].

From the simple facts recorded in IV.2 we obtain the following:

THEOREM IV.3. Suppose $E^{\prime}$ or $F^{\prime}$ has the approximation property. Then

(d) $T \in \mathrm{FN}(E, F)$ if and only if $T^{\prime} \in \mathrm{CN}\left(F^{\prime}, E^{\prime}\right)$; and

(e) if $E$ is reflexive, $T \in \mathrm{CN}(E, F)$ if and only if $T^{\prime} \in \mathrm{FN}\left(F^{\prime}, E^{\prime}\right)$.

Proof. (d) If $T \in \mathrm{FN}(E, F)$ then $T_{a}$, hence $\left[T_{a}\right]^{\prime}$, is nuclear. By (a), $\left[T_{a}\right]^{\prime}=\left[T^{\prime}\right]_{K}$, i.e. $T^{\prime} \in \mathrm{CN}\left(F^{\prime}, E^{\prime}\right)$. Now suppose $T^{\prime} \in \mathrm{CN}\left(F^{\prime}, E^{\prime}\right)$, i.e. $\left[T^{\prime}\right]_{K}$ is nuclear. Again by (a), $\left[T_{a}\right]^{\prime}$ is nuclear. Since $E^{\prime}$ or $F^{\prime}$ has the approximation property it follows [7] that $T_{a}$ is nuclear, i.e. $T \in \mathrm{FN}(E, F)$. The proof of (e) is analogous using IV.2(c).

REMARK IV.4. In IV.3 we used the approximation property only to infer that if $T^{\prime}$ is nuclear then $T$ is nuclear. Whether this is true in general is not known and apparently difficult.

Indeed, let us say that a Banach space $E$ has the nuclear adjoint property $(N A P)$, if $T \in \mathscr{L}(E, F)$ and $T^{\prime} \in \mathrm{N}\left(F^{\prime}, E^{\prime}\right)$ implies $T \in \mathrm{N}(E, F)$ for every Banach space $F$. The result of Grothendieck used above can now be stated: If $E^{\prime}$ has the approximation property then $E$ has NAP. Thus one sees the difficulties in trying to avoid the approximation property hypothesis in IV.3.

3. The linear and "ideal" structure of $\mathrm{CN}(E, F)$. From IV.2(a) we see that $\left([T+S]_{a}\right)^{\prime}=\left([T+S]^{\prime}\right)_{K}=\left[S^{\prime}+T^{\prime}\right]_{K}$ and so, by II.6, CN $(E, F)$ is not in general a linear space. As to be expected the ideal structure is also pathological.

Theorem IV.4. If $T \in \mathscr{L}(E, F), S \in \mathrm{CN}(F, G)$ then $S T \in \mathrm{CN}(E, G)$ for any Banach spaces $E, F$ and $G$.

Proof. The operator $\hat{T}: E / \operatorname{Ker} S T \rightarrow F / \operatorname{Ker} S$ defined by $\hat{T}(x+\operatorname{Ker} S T)=T x+$ Ker $S$ is well defined, continuous (and one-to-one). Consider the following diagram:

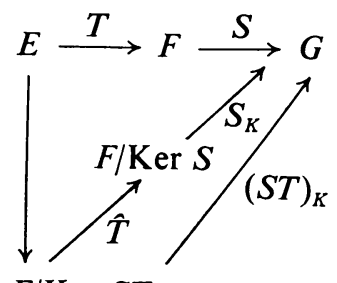

$E / \operatorname{Ker} S T$ 
where the unnamed maps are canonical. Clearly $(S T)_{K}=S_{K} \hat{T}$. By hypothesis $S_{K}$ is nuclear and thus $(S T)_{K}$ is nuclear as asserted.

On the other hand if $T \in \mathrm{CN}(E, F)$ and $S \in \mathscr{L}(F, G)$ then, in general, $S T \notin$ $\mathrm{CN}(E, G)$. This results from the fact that every nuclear operator admits a completely nuclear lifting. This fact is proved in Chapter V.

4. The conjecture of Grothendieck. In this section we prove the following theorem:

TheOREM IV.5. Suppose $\mathscr{L}(E, F)=\mathrm{CN}(E, F)$ and $E$ is isomorphic to a conjugate space. Then one of $E, F$ is finite dimensional.

Proof. Suppose $E=X^{\prime}$ for some Banach space $X$ and that $\operatorname{dim} E, \operatorname{dim} F=\infty$. By Theorem I.15, $E$ has a sufficiently Euclidean factor space, say $G$, and by Theorem I.21 a nonabsolutely summing operator $T$ from $G$ to $F$. If $G=E / Z$ then clearly $Z \subset \operatorname{Ker} T \varphi$ where $\varphi$ is the quotient map. By hypothesis $T \varphi$ is completely nuclear. Thus we have

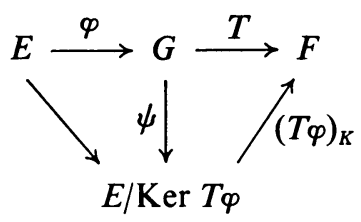

where $\psi(g)=\psi(e+Z)=e+\operatorname{Ker} T \varphi$. Clearly $T=(T \varphi)_{K} \psi$ and so $T$ must be nuclear. But $T$ is not even absolutely summing.

Theorem IV.5 is also true under the hypothesis that $E^{\prime}$ contains an infinitedimensional reflexive subspace.

5. A characterization of Hilbert space II. As in the case of $\mathrm{FN}(E, F)$, there are important cases when $\mathrm{CN}(E, F)$ is a linear space.

THeorem IV.6. Suppose $E^{\prime}$ has the approximation property and $T \in \mathrm{N}(E, F)$. If $E$ is reflexive and $\mathrm{cl}\left(T^{\prime}\left(F^{\prime}\right)\right)$ is complemented in $E^{\prime}$ then $T \in \mathrm{CN}(E, F)$.

Proof. If the hypotheses are satisfied then $\left[T^{\prime}\right]_{a}$ is nuclear and hence $\left[T_{K}\right]^{\prime}$ is nuclear; since $E^{\prime}$ has the approximation property $T_{K}$ is nuclear, i.e. $T \in \mathrm{CN}(E, F)$.

COROLlaRY IV.7. If $E$ is isomorphic to a Hilbert space (in particular if $\operatorname{dim} E$ $<+\infty)$ then, for any Banach space $F, \mathrm{~N}(E, F)=\mathrm{CN}(E, F)$.

THEOREM IV.8. Suppose $E$ is reflexive and

(i) each closed subspace $G$ of $E^{\prime}$ has the approximation property, and

(ii) $\mathrm{N}(E, F)=\mathrm{CN}(E, F)$ for all Banach spaces $F$.

Then every closed subspace of $E^{\prime}$ is complemented.

Proof. Let $T \in \mathrm{N}\left(F, E^{\prime}\right)$ where $F$ is an arbitrary Banach space. Then

$$
T^{\prime} \in \mathrm{N}\left(E^{\prime \prime}, F^{\prime}\right)=\mathrm{N}\left(E, F^{\prime}\right)
$$


since $E$ is reflexive. Since $\mathrm{N}\left(E, F^{\prime}\right)=\mathrm{CN}\left(E, F^{\prime}\right),\left[T^{\prime}\right]_{K}$ is nuclear and so $\left(T_{a}\right)^{\prime}$ is nuclear and as in the previous results $T_{a}$ is nuclear. Thus $\mathrm{N}\left(F, E^{\prime}\right)=\mathrm{FN}\left(F, E^{\prime}\right)$ for each Banach space $F$. By II.9 every subspace of $E^{\prime}$ is complemented.

We now prove the completely nuclear analog of II.11.

THEOREM IV.9. Suppose $E^{\prime}$ is as in IV.8 and the canonical operator $\mathrm{N}\left(F, E^{\prime}\right) \rightarrow$ $\mathrm{CN}\left(F, E^{\prime}\right)$ (i.e. $T \rightarrow T_{K}$ ) is an isometry for each Banach space $F$. Then $E$ is isometrically isomorphic to a Hilbert space.

Proof. The hypotheses imply those of II.11 for $\mathrm{N}\left(F, E^{\prime}\right)$ and so $E^{\prime}$, hence $E$, is isometrically isomorphic to a Hilbert space.

REMARK IV.10. From the above results and those of Chapter II we have $\mathrm{N}(H, H)=\mathrm{FN}(H, H)=\mathrm{CN}(H, H)$ for any Hilbert space $H$. Modulo the restrictions of II.11 and III.9 the converse is also true.

\section{An analog of a conjecture of Grothendieck II.}

Remark IV.11. There is a completely nuclear operator $T$ such that $T^{\prime}$ is not completely nuclear.

Indeed the example of II.13 suffices. For if $T$ is the operator of II.13, $T \in \mathrm{N}\left(l_{2}, l_{1}\right)$; by IV.9, $T \in \mathrm{CN}\left(l_{2}, l_{1}\right)$. If $T^{\prime}$ were completely nuclear then $\left(T^{\prime}\right)_{K}$ hence $\left(T_{a}\right)^{\prime}$ would be nuclear. Since all spa $s$ under consideration (and their adjoints) have the approximation property, $\mathrm{FN}\left(l_{2}, l_{1}\right)$ which contradicts II.13.

Observe that if $S=T^{\prime}$ above then $S$ is an operator such that $S^{\prime}$ is completely nuclear and $S$ is not completely nuclear.

7. Questions. Although there are many questions concerning completely nuclear operators we list here only some of the obvious ones.

(Q7) Is IV.5 true if $E$ is not necessarily isomorphic to a conjugate space?

We suspect that the answer to $(\mathrm{Q} 7)$ is affirmative. A positive answer would come from an affirmative answer to

(Q8) Does every infinite-dimensional Banach space have an infinite-dimensional sufficiently Euclidean factor space?

(Q8) appears to be difficult.

Also we do not know if IV.3(e) is true if the reflexivity hypothesis is dropped. This problem is equivalent to (by IV.3(d)) the following:

(Q9) If $T$ is completely nuclear is $T^{\prime \prime}$ completely nuclear? Conversely?

The difficulties of (Q9) relate to those of the problem of Grothendieck: If $T^{\prime}$ is nuclear, is $T$ nuclear? Indeed we have

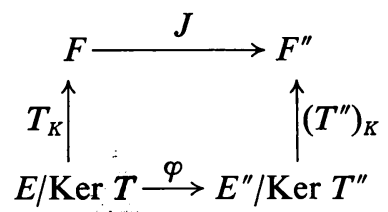

where $J$ is evaluation and $\varphi(e+\operatorname{Ker} T)=e+\operatorname{Ker} T^{\prime \prime}$ (viewing $e$ as an element of $E^{\prime \prime}$ ). If $T_{K}$ is nuclear (i.e. $T$ is completely nuclear) then it follows that $\left(T^{\prime \prime}\right)_{K} \varphi$ is nuclear 
but, in general, there appears to be no way to conclude that $\left(T^{\prime \prime}\right)_{K}$ is nuclear. Likewise even though $J$ is an isometry there is no way to guarantee that $T_{K}$ is nuclear knowing that $J T_{K}$ is nuclear. Of course assuming that $E^{\prime \prime}$ has the approximation property yields an affirmative answer to (Q9) but in general we do not see how to overcome the reflexivity hypothesis.

\section{Characterizations of $\mathscr{L}_{1}$-Spaces}

1. $\mathscr{L}_{1}$-spaces. This chapter provides the dual of Theorem III.3. We begin with

THEOREM V.1. The following assertions are equivalent:

(i) $E$ is an $\mathscr{L}_{1}$-space;

(ii) $\mathrm{N}(F, E)=\mathrm{CN}(F, E)$ for every Banach space $F$;

(iii) for every Banach space $F$ and closed subspace $F_{0}$ of $F$, the canonical map $F_{0} \hat{\otimes} E \rightarrow F \hat{\otimes} E$ is an isomorphism; and

(iv) $E^{\prime}$ is an injective space.

Proof. That (i) and (iv) are equivalent is the result of Lindenstrauss and Rosenthal [19].

Let $J$ be the canonical map from $F_{0} \hat{\otimes} E \rightarrow F \hat{\otimes} E$ where $F_{0}$ is a closed subspace of the arbitrary Banach space $F$. Clearly $J$ is an isomorphism if and only if $J^{\prime}$, which is restriction $\mathscr{L}\left(F, E^{\prime}\right) \rightarrow \mathscr{L}\left(F_{0}, E^{\prime}\right)$, is onto. The latter happens if and only if $E^{\prime}$ is injective. Thus (i), (iii) and (iv) are equivalent.

(i) $\rightarrow$ (ii). As remarked in III.4 an $\mathscr{L}_{\infty}$-space has the approximation property. By [19] and [7] so does an $\mathscr{L}_{1}$-space and thus $E$ has NAP (IV.4). Thus if $T \in \mathrm{N}(F, E)$, $T^{\prime} \in \mathrm{FN}\left(E^{\prime}, F^{\prime}\right)$ by III.2(iii). From the last paragraph of Chapter IV it follows that $T$ is completely nuclear.

(ii) $\rightarrow$ (i). Suppose $T \in \mathrm{N}(F, E)=\mathrm{CN}(F, E)$ and let $K=\operatorname{Ker} T$. Let $F_{0}$ be a closed subspace of $F$. If $J$ denotes the canonical map $F_{0} \hat{\otimes} E \rightarrow F \hat{\otimes} E$ then $J \mid K$ is closed. Thus we obtain the diagram

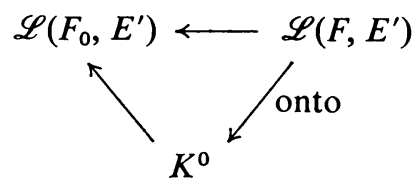

It is easily checked that $K^{0}$ contains the operators $\mathrm{C}$ finite rank from $F_{0}$ to $E^{\prime}$. As in III.3 it follows that $E^{\prime}$ is an $\mathscr{L}_{\infty}$-space. Thus by '19] $E$ is an $\mathscr{L}_{1}$-space.

Remark V.2. (i) Grothendieck [7] observe that for $E$ an $L_{1}(\mu)$-space $F_{0} \widehat{\otimes} E$ $\rightarrow F \hat{\otimes} E$ is always an isometry (see also [30]). It follows from the results of [37] that this above property actually characterizes $L_{1}(\mu)$-spaces. This first led us to believe that V.1(iii) indeed characterized $\mathscr{L}_{1}$-spaces.

Since there are $\mathscr{L}_{1}$-spaces which are not $L_{1}(\mu)$-spaces [16], [18], V.1 completes the study begun by Grothendieck, i.e. the isometric and isomorphic characterizations are now complete. 
(ii) We should mention that in view of III.3 and V.1 and the remark, it seems reasonable to suspect that if one assumes that $E$ has the metric approximation property that III.3(iii) implies that $E^{\prime \prime}$ is a $P_{1}$-space. However, an example of Isbell and Semadeni [38] shows that this is not the case.

(iii) Again in view of Theorem III.3 it is natural to consider spaces $E$ for which $\mathrm{I}(F, E)=\mathrm{N}(F, E)$ for any Banach space $F$. However by 0.15 , this is always true if $E$ is reflexive. However, it is interesting that this property characterizes the $l_{1}(\Gamma)$-spaces among the $L_{1}(\mu)$-spaces. Indeed by [7, Proposition 9, p. 64] an $l_{1}(\Gamma)-$ space has this property. Since $L_{\infty}[0,1] \rightarrow L_{1}[0,1]$ is integral and not nuclear it follows by a standard decomposition argument that this is true for any nonatomic measure $\mu$.

2. Corollaries. There are corollaries to V.1 analogous to those of III.3.

COROLlaRY V.3. Let $E$ be an arbitrary Banach space and $F$ a subspace of an $\mathscr{L}_{1}$-space. Then there is a $T: E \rightarrow F$ which is not nuclear.

Proof. Construct $T=\sum_{j} \sum_{\sigma_{j}} \lambda_{i j} f_{i j} \otimes y_{i j}$ as in I.22 (in particular [ $\left.f_{i j}\right]$ is sufficiently Euclidean) and suppose $J: F \rightarrow G$ is an into isomorphism where $G$ is an $\mathscr{L}_{1}$-space. If $T$ were nuclear then $T^{\prime} J^{\prime}$ would be nuclear and since the domain of $T^{\prime} J^{\prime}$ is an $\mathscr{L}_{\infty}$-space, the astriction of $T^{\prime} J^{\prime}$ would be nuclear. But the image of $T^{\prime} J^{\prime}$ is $\left[f_{i j}\right]$ and it is easy to see that the adjoint of the astriction of $T^{\prime} J^{\prime}$ is not nuclear. Thus $T$ is not nuclear.

Our next result is the analog of III.9.

COROLLARY V.4. Every nuclear operator admits a completely nuclear lifting.

Proof. Let $T: E \rightarrow F$ be nuclear where $E$ and $F$ are arbitrary Banach spaces. It will be known that $F$ is isomorphic to $l_{1}(\Gamma) / Z$ for suitable $\Gamma$ and $Z$ and, by 0.17 , $T$ has a nuclear lifting $\tilde{T}$ to $l_{1}(\Gamma)$

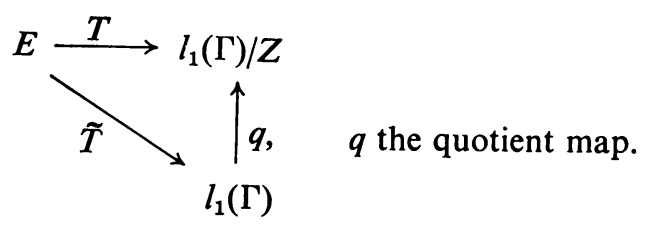

By V.1(ii), $\tilde{T}$ is completely nuclear.

The analog of III.10 is just Grothendieck's "strong" factorization of a nuclear operator. Indeed we know from 0.14 that if $T$ is nuclear, $T$ factors through $c_{0} \rightarrow l_{1}$ and this latter map is nuclear. Thus Grothendieck's result together with III.3 and V.1 shows that every nuclear operator factors through an operator that is both fully nuclear and completely nuclear. 
3. Questions. Even in view of V.2(iii) there is hope that an analog of III.7 is true: Indeed

(Q10) Suppose $E$ is nonreflexive and has an unconditional basis. If $\mathrm{I}(F, E)$ $=\mathrm{N}(F, E)$ for each Banach space $F$, is $E$ isomorphic to $l_{1}$ ?

We know only a little about the situation of (Q10).

Grothendieck has observed that the injection $l_{1} \rightarrow c_{0}$ is integral [8]. Since this map is not compact it is surely not nuclear. By a result of R. C. James [12], if $E$ satisfies the first hypothesis of (Q10) then $E$ must contain either $c_{0}$ or $l_{1}$. The second hypothesis and the above remark show that $E$ cannot contain $c_{0}$. Indeed, since $E$ is separable it follows from a result of Sobczyk [32] that if $c_{0} \subset E$ then $c_{0}$ is complemented in $E$. Thus if $l_{1} \rightarrow c_{0} \subset E$ were nuclear, $l_{1} \rightarrow c_{0}$ would be nuclear.

\section{SUMmaRY}

1. The $\mathscr{L}_{\infty}$ - and $\mathscr{L}_{1}$-spaces. As mentioned in the introduction the $\mathscr{L}_{\infty}$ - and $\mathscr{L}_{1}$-spaces appear at this developing stage to play the role in the study of the isomorphic structure of Banach spaces that is played by the $L_{\infty}(\mu)$ - and $L_{1}(\mu)$-spaces in the study of the isometric structure of such spaces. The important papers [18], [19] have solved many of the problems concerning $\mathscr{L}_{p}$-spaces $(1 \leqq p \leqq \infty)$ but there are still many unsolved problems (see e.g. [19]).

Our attempt in this work has been to give some internal characterizations of the $\mathscr{L}_{\infty}$ - and $\mathscr{L}_{1}$-spaces and, of course, we have relied heavily on the papers [18] and [19]. To conclude this work it seems worthwhile to summarize what is now known about the $\mathscr{L}_{\infty}$ - and $\mathscr{L}_{1}$-spaces. All statements not contained in this paper are from [18] and [19]. In what follows $K(E, F)$ denotes the compact operators from $E$ to $F$.

The $\mathscr{L}_{\infty}$-spaces.

THEOREM VI.1. The following are equivalent:

(a) $E$ is an $\mathscr{L}_{\infty}$-space;

(b) $E^{\prime}$ is an $\mathscr{L}_{1}$-space;

(c) $E^{\prime \prime}$ is injective;

(Extension properties)

(d) for each pair of Banach spaces, $Z, Y$ with $Z \supset Y$, every $T \in K(Y, E)$ has an extension $\tilde{T} \in K(Z, X)$;

(e) same as (d) but $\tilde{T} \in \mathscr{L}(Z, X)$;

(f) for Banach spaces $Y$ and $Z \supset E$, every $T \in K(E, Y)$ has an extension $\tilde{T} \in K(Z, Y)$;

(g) same as (f) except $\tilde{T} \in \mathscr{L}(Z, Y)$;

(h) there is a $\lambda>0$ such that for all pairs of Banach spaces $Y, Z, Z \supset Y, \operatorname{dim} Z$ $<+\infty$, each $T \in \mathscr{L}(Y, E)$ has an extension $\tilde{T} \in \mathscr{L}(Z, E)$ with $\|\tilde{T}\| \leqq \lambda\|T\|$;

(i) there is a $\lambda>0$ such that if $\operatorname{dim} Y<+\infty, Z \supset E$; each $T \in \mathscr{L}(E, Y)$ has an extension $\tilde{T} \in \mathscr{L}(Z, Y)$ with $\|\tilde{T}\| \leqq \lambda\|T\|$.

(Operator properties)

(j) $\mathrm{I}(E, F)=\mathrm{AS}(E, F)$ for all Banach spaces $F$; 
(k) $\mathrm{N}(E, F)=\mathrm{QN}(E, F)$ for all Banach spaces $F$;

(l) $\mathrm{N}(E, F)=\mathrm{FN}(E, F)$ for all Banach spaces $F$ (for all conjugate Banach spaces $F)$ (for all reflexive Banach spaces $F$ );

(m) $\mathrm{I}(E, F)=\mathrm{FI}(E, F)$ for all Banach spaces $F$ (for all conjugate Banach spaces $F)$ (for all reflexive Banach spaces $F$ );

(n) analogous to $(\mathrm{j})$ or $(\mathrm{k})$ with $E$ the range space.

THEOREM VI.2. (a) A complemented subspace of an $\mathscr{L}_{\infty}$-space is an $\mathscr{L}_{\infty}$-space;

(b) iffor all pairs of Banach spaces $Y, Z$ with $Z \supset Y$, the restriction map $r: \mathscr{L}(Z, E)$ $\rightarrow \mathscr{L}(Y, E)$ has a closed range then $E$ is an $\mathscr{L}_{\infty}$-space.

The $\mathscr{L}_{1}$-spaces.

THEOREM VI.3. The following are equivalent:

(a) $E$ is an $\mathscr{L}_{1}$-space;

(b) $E^{\prime}$ is an $\mathscr{L}_{\infty}$-space;

(c) $E^{\prime}$ is injective;

(Lifting properties)

(d) for all pairs of Banach spaces $Z, Y$ and onto operators $\varphi: Z \rightarrow Y$, each $T \in K(E, Y)$ has a lifting $\tilde{T} \in K(E, Z)$;

(e) same as (d) but with $\tilde{T} \in \mathscr{L}(E, Z)$;

(f) for all pairs of Banach spaces $Y, Z$ and onto operators $\varphi: Z \rightarrow E$, every $T \in K(Y, E)$ has a lifting $\tilde{T} \in K(Y, Z)$;

(g) same as (f) with $\tilde{T} \in \mathscr{L}(Y, Z)$;

(h) there is a $\beta>0$ such that for all pairs of Banach spaces $Y, Z$, and onto operators $\varphi: Z \rightarrow Y, \operatorname{dim} Z<+\infty$, each $T \in \mathscr{L}(E, Y)$ has a lifting $\tilde{T} \in K(E, Z)$ with $\|\tilde{T}\| \leqq \beta\|T\| ;$

(i) there is a $\beta>0$ such that if $\operatorname{dim} Y<+\infty$ and $\varphi: Z \rightarrow E$ is onto, each $T \in \mathscr{L}(Y, E)$ has a lifting $\tilde{T} \in \mathscr{L}(Y, Z)$ with $\|\tilde{T}\| \leqq \beta\|T\|$;

(Operator properties)

(j) $\mathrm{N}(F, E)=\mathrm{CN}(F, E)$ for every Banach space $F$; and

(k) for every pair of Banach spaces $Y, Z, Z \supset Y$, the canonical operator $Y \hat{\otimes} E$ $\rightarrow Z \hat{\otimes} E$ is an isomorphism.

THEOREM VI.4. (a) Every $\mathscr{L}_{1}$-space is isomorphic to a subspace of an $L_{1}(\mu)$-space for some measure $\mu$;

(b) a complemented subspace of an $\mathscr{L}_{1}$-space is an $\mathscr{L}_{1}$-space; and

(c) if $E$ is an $L_{1}(\mu)$-space and $\mathrm{I}(F, E)=\mathrm{N}(F, E)$ for all Banach spaces $F$ then $E$ is an $l_{1}(\Gamma)$ for some $\Gamma$.

2. Final remarks. We conclude this paper by remarking that there is a theory of fully- $p$-integral, fully- $p$-nuclear, completely- $p$-integral, completely- $p$-nuclear, etc. which, roughly speaking, parallels the development of [41]. Indeed we have not mentioned the multiplication theory of [41] at all and have only briefly mentioned the duality theory for the operators defined in this work. It is possible to character- 
ize the $\mathscr{L}_{p}$-spaces using these concepts. Some of the results we have obtained seem to us, at present, to be artificial and we have hopes of significantly improving them. These results will appear in a later paper.

\section{BIBLIOGRAPHY}

1. S. Banach, Théorie des opérations linéaires, Monografie Mat., PWN, Warsaw, 1932; reprint, Chelsea, New York, 1955. MR 17, 175.

2. S. Banach and S. Mazur, Zur Theorie der linearen Dimension, Studia Math. 4 (1933), 100-112.

3. P. Civin and B. Yood, Quasi-reflexive spaces, Proc. Amer. Math. Soc. 8 (1957), 906-911. MR 19, 756.

4. A. Dvoretzky, Some results on convex bodies and Banach spaces, Proc. Internat. Sympos. Linear Spaces (Jerusalem, 1960), Jerusalem Academic Press, Jerusalem, 1960, pp. 123-160. MR 25 \#2518.

5. A. Dvoretzky and C. A. Rogers, Absolute and unconditional convergence in normed linear spaces, Proc. Nat. Acad. Sci. U.S.A. 36 (1950), 192-197. MR 11, 525.

6. M. M. Grinblyum, On the representation of a space of type $B$ in the form of a direct sum of subspaces, Dokl. Akad. Nauk SSSR 70 (1950), 749-752. MR 11, 525.

7. A. Grothendieck, Produits tensoriels topologiques et espaces nucléaires, Mem. Amer. Math. Soc. No. 16 (1955). MR 17, 763.

8. —_ Résumé de la théorie métrique des produits tensoriels topologiques, Bol. Soc. Mat. São Paulo 8 (1953), 1-79. MR 20 \#1194.

9. — - Sur certaines classes de suites dans les espaces de Banach, et le théorème de Dvoretzky-Rogers, Bol. Soc. Mat. São Paulo 8 (1953), 81-110. MR 20 \#1195.

10. B. Grünbaum, Projection constants, Trans. Amer. Math. Soc. 95 (1960), 451-465. MR 22 \#4937.

11. V. I. Gurariǐ, Inclinations of subspaces and conditional bases in Banach space, Dokl. Akad. Nauk SSSR 145 (1962), 504-506=Soviet Math. Dokl. 3 (1962), 1028-1030. MR 27 \#2835.

12. R. C. James, Bases and reflexivity of Banach spaces, Ann. of Math. (2) 52 (1950), 518-527. MR 12, 616.

13. S. Kaczmarz and H. Steinhaus, Theorie der Orthogonalreihen, Monografie Mat., Tom 6, PWN, Warsaw, 1935; reprint, Chelsea, New York, 1951. MR 20 \#1148.

14. S. Kakutani, Some characterizations of Euclidean space, Japan. J. Math. 16 (1939), 93-97.

15. J. Lindenstrauss, Extension of compact operators, Mem. Amer. Math. Soc. No. 48 (1964). MR 31 \#3828.

16. - On a certain subspace of $l_{1}$, Bull. Acad. Polon. Sci. Sér. Sci. Math. Astronom. Phys. 12 (1964), 539-542. MR 30 \#5153.

17. - On the modulus of smoothness and divergent series in Banach spaces, Michigan Math. J. 10 (1963), 241-252. MR 29 \#6316.

18. J. Lindenstrauss and A. Pełczyński, Absolutely summing operators in $L_{p}$-spaces and their applications, Studia Math. 29 (1968), 275-326. MR 37 \#6743.

19. J. Lindenstrauss and H. P. Rosenthal, The $L_{p}$-spaces, Israel J. Math. 7 (1969), 325-349.

20. A. Miljutin, Isomorphism of the spaces of continuous functions over compact sets of the cardinality of the continuum, Teor. Funkciǐ Funkcional. Anal. i Priložen. No. 2 (1966), 150-156. (Russian) MR 34 \#6513.

21. A. Pełczyński, Projections in certain Banach spaces, Studia Math. 19 (1960), 209-228. MR 23 \#A3441. 
22. A. Pełczyński and M. I. Kadec, Bases, lacunary sequences and complemented subspaces in the spaces $L_{p}$, Studia Math. 21 (1962), 161-176.

23. A. Pietsch, Nukleare lokalkonvexe Räume, Akademie-Verlag, Berlin, 1965. MR 31 \#6114.

24. — Absolut p-summierende Abbildungen in normierten Räumen, Studia Math. 28 (1967), 333-353.

25. - Q Quasinukleare Abbildungen in normierten Räumen, Math. Ann. 165(1966), 76-90.

26. H. P. Rosenthal, Projections onto translation-invariant subspaces of $L^{p}(G)$, Mem. Amer. Math. Soc. No. 63 (1966). MR 35 \#2080.

27. - On complemented and quasi-complemented subspaces of quotients of $C(S)$ for Stonian S, Proc. Nat. Acad. Sci. U.S.A. 60 (1968), 1165-1169. MR 37 \#6740.

28. D. Rutovitz, Some parameters associated with finite-dimensional Banach spaces, J. London Math. Soc. 40 (1965), 241-255. MR 32 \#8120.

29. H. H. Schaefer, Topological vector spaces, Macmillan, New York, 1966. MR 33 \#1689.

30. L. Schwartz, Produits tensoriels topologiques d'espaces vectoriels topologiques, Séminaire Schwartz de la Faculté des Sciences de Paris, 1953/54, Secrétariat mathématique, Paris, 1954. MR 17, 764 .

31. A. Sobczyk, Projections in Minkowski and Banach spaces, Duke Math. J. 8 (1941), 78-106. MR 2, 220.

32. - Projection of the space $(m)$ on its subspace $\left(c_{0}\right)$, Bull. Amer. Math. Soc. 47 (1941), 938-947. MR 3, 205.

33. W. Wilson, On the selection of basic sequences in Banach spaces, Thesis, Louisiana State University, Baton Rouge, 1969.

34. M. Zippin, H. P. Rosenthal and W. B. Johnson, On bases, finite dimensional decompositions and weaker structures in Banach spaces, Israel J. Math. 9 (1971), 488-506.

35. M. M. Day, Normed linear spaces, Springer-Verlag, Berlin, 1958. MR 20 \#1187.

36. D. B. Goodner, Projections in normed linear spaces, Trans. Amer. Math. Soc. 69 (1950), 89-108. MR 12, 266.

37. A. Grothendieck, Sur les applications linéaires faiblement compactes d'espaces du type $C(K)$, Canad. J. Math. 5 (1953), 129-173. MR 15, 438.

38. J. R. Isbell and Z. Semadeni, Projection constants and spaces of continuous functions, Trans. Amer. Math. Soc. 107 (1963), 38-48. MR 26 \#4169.

39. G. Köthe, Hebbare lokalkonvexe Räume, Math. Ann. 165 (1966), 181-195. MR 33 \#4651.

40. S. Kwapień, Some remarks on $(p, q)$-absolutely summing operators in $L_{p}$-spaces, Studia Math. 29 (1968), 327-337. MR 37 \#6767.

41. A. Pietsch and A. Perrson, p-nukleare and p-integrale Abbildungen in Banachräumen, Studia Math. 33 (1969), 19-62. MR 39 \#4645.

42. (a) C. P. Stegall, Operator characterizations of $\mathscr{L}_{\infty}$-and $\mathscr{L}_{1}$-spaces, Bull. Polon. Acad. Sci. 19 (1971), 73-75.

(b) C. P. Stegall and J. R. Retherford, Fully nuclear operators, Bull. Amer. Math. Soc. 76 (1970), 1077-1081.

43. A. E. Taylor, Introduction to functional analysis, Wiley, New York, 1961.

Department of Mathematics, Louisiana State University, Baton Rouge, Louisiana 70803

Current address (Stegall): Department of Mathematics, State University of New York, Binghamton, New York 13901 